\title{
The synergistic effect of concatenation in phylogenomics: the case in Pantoea
}

\author{
Marike Palmer ${ }^{1}$, Stephanus N Venter ${ }^{\text {Corresp., }}{ }^{1}$, Alistair R McTaggart ${ }^{1,2}$ ， Martin P A Coetzee ${ }^{1}$, Stephanie Van \\ Wyk $^{1}$, Juanita R Avontuur ${ }^{1}$, Chrizelle W Beukes ${ }^{1}$, Gerda Fourie ${ }^{1}$, Quentin C Santana ${ }^{1}$, Magriet A Van Der \\ Nest $^{1}$, Jochen Blom ${ }^{3}$, Emma T Steenkamp ${ }^{1}$ \\ 1 Department of Biochemistry, Genetics and Microbiology, DST-NRF Centre of Excellence in Tree Health Biotechnology (CTHB) and Forestry and \\ Agricultural Biotechnology Institute (FABI), University of Pretoria, Pretoria, Gauteng, South Africa \\ 2 Queensland Alliance for Agriculture and Food Innovation, University of Queensland, Brisbane, Queensland, Australia \\ 3 Bioinformatics and Systems Biology, Justus Liebig Universität Gießen, Giessen, Germany \\ Corresponding Author: Stephanus N Venter \\ Email address: fanus.venter@up.ac.za
}

With the increased availability of genome sequences for bacteria, it has become routine practice to construct genome-based phylogenies. These phylogenies have formed the basis for various taxonomic decisions, especially for resolving problematic relationships between taxa. Despite the popularity of concatenating shared genes to obtain wellsupported phylogenies, various issues regarding this combined-evidence approach have been raised. These include the introduction of phylogenetic error into datasets, as well as incongruence due to organism-level evolutionary processes, particularly horizontal gene transfer and incomplete lineage sorting. Because of the huge effect that this could have on phylogenies, we evaluated the impact of phylogenetic conflict caused by organism-level evolutionary processes on the established species phylogeny for Pantoea, a member of the Enterobacterales. We explored the presence and distribution of phylogenetic conflict at the gene partition and nucleotide levels, by identifying putative inter-lineage recombination events that might have contributed to such conflict. Furthermore, we determined whether smaller, randomly constructed datasets had sufficient signal to reconstruct the current species tree hypothesis or if they would be overshadowed by phylogenetic incongruence. We found that no individual gene tree was fully congruent with the species phylogeny of Pantoea, although many of the expected nodes were supported by various individual genes across the genome. Evidence of recombination was found across all lineages within Pantoea, and provides support for organism-level evolutionary processes as a potential source of phylogenetic conflict. The phylogenetic signal from at least 70 random genes recovered robust, well-supported phylogenies for the backbone and most species relationships of Pantoea, and was unaffected by phylogenetic conflict within the dataset. Furthermore, despite providing limited resolution among taxa at the level of single gene trees, concatenated analyses of genes that were identified as having no signal resulted in 
a phylogeny that resembled the species phylogeny of Pantoea. This distribution of signal and noise across the genome presents the ideal situation for phylogenetic inference, as the topology from $a \geq 70$-gene concatenated species phylogeny is not driven by single genes, and our data suggests that this finding may also hold true for smaller datasets. We thus argue that, by using a concatenation-based approach in phylogenomics, one can obtain robust phylogenies due to the synergistic effect of the combined signal obtained from multiple genes. 


\section{The synergistic effect of concatenation in phylogenomics: the case in}

\section{Pantoea}

3 Marike Palmer ${ }^{1}$, Stephanus N. Venter ${ }^{1}$, Alistair R. McTaggart 1,2, Martin P. A. Coetzee ${ }^{1}$,

4 Stephanie van Wyk ${ }^{1}$, Juanita R. Avontuur ${ }^{1}$, Chrizelle W. Beukes ${ }^{1}$, Gerda Fourie ${ }^{1}$, Quentin C.

5 Santana ${ }^{1}$, Magriet A. van der Nest ${ }^{1}$, Jochen Blom ${ }^{3}$, Emma T. Steenkamp ${ }^{1}$

6 1. Department of Biochemistry, Genetics and Microbiology, DST-NRF Centre of Excellence in

7 Tree Health Biotechnology (CTHB) and Forestry and Agricultural Biotechnology Institute

8 (FABI), University of Pretoria, Pretoria, Gauteng, South Africa

9 2. Queensland Alliance for Agriculture and Food Innovation, University of Queensland, Brisbane,

10 Queensland, Australia

11 3. Bioinformatics and Systems Biology, Justus Liebig Universität Gießen, Giessen, Germany

13 Corresponding author:

14 Stephanus N. Venter

E-mail address: fanus.venter@up.ac.za 


\section{Abstract}

With the increased availability of genome sequences for bacteria, it has become routine practice to construct genome-based phylogenies. These phylogenies have formed the basis for various taxonomic decisions, especially for resolving problematic relationships between taxa. Despite the popularity of concatenating shared genes to obtain well-supported phylogenies, various issues regarding this combined-evidence approach have been raised. These include the introduction of phylogenetic error into datasets, as well as incongruence due to organism-level evolutionary processes, particularly horizontal gene transfer and incomplete lineage sorting. Because of the huge effect that this could have on phylogenies, we evaluated the impact of phylogenetic conflict caused by organism-level evolutionary processes on the established species phylogeny for Pantoea, a member of the Enterobacterales. We explored the presence and distribution of phylogenetic conflict at the gene partition and nucleotide levels, by identifying putative inter-lineage recombination events that might have contributed to such conflict. Furthermore, we determined whether smaller, randomly constructed datasets had sufficient signal to reconstruct the current species tree hypothesis or if they would be overshadowed by phylogenetic incongruence. We found that no individual gene tree was fully congruent with the species phylogeny of Pantoea, although many of the expected nodes were supported by various individual genes across the genome. Evidence of recombination was found across all lineages within Pantoea, and provides support for organism-level evolutionary processes as a potential source of phylogenetic conflict. The phylogenetic signal from at least 70 random genes recovered robust, well-supported phylogenies for the backbone and most species relationships of Pantoea, and was unaffected by phylogenetic conflict within the dataset. Furthermore, despite providing limited resolution among taxa at the level of single gene trees, concatenated analyses 
39 of genes that were identified as having no signal resulted in a phylogeny that resembled the

40 species phylogeny of Pantoea. This distribution of signal and noise across the genome presents

41 the ideal situation for phylogenetic inference, as the topology from a $\geq 70$-gene concatenated

42 species phylogeny is not driven by single genes, and our data suggests that this finding may also

43 hold true for smaller datasets. We thus argue that, by using a concatenation-based approach in

44 phylogenomics, one can obtain robust phylogenies due to the synergistic effect of the combined 45 signal obtained from multiple genes. 


\section{Introduction}

Whole genome sequences are now routinely used for phylogenetic inference, particularly in bacteria (Abdul Rahman et al. 2016; Beukes et al. 2017; Callister et al. 2008; Gupta et al. 2015; Meehan \& Beiko 2014; Palmer et al. 2017; Schwartz et al. 2015; Zhang et al. 2011). Many approaches for investigating evolutionary relationships across different taxonomic ranks have been developed (Daubin \& Gouy 2001; Daubin et al. 2002; Jolley et al. 2012; Yokono et al. 2018). These range from alignment-free approaches (Yokono et al. 2018) to alignment-based analyses of a small number of highly conserved genes across large numbers of taxa [e.g. different bacterial phyla or orders (Abdul Rahman et al. 2016; Gupta et al. 2015; Jolley et al. 2012)], to using hundreds or thousands of genes, obtained from whole genome sequences and shared by all members of smaller groups [e.g. species, genus or family (Meehan \& Beiko 2014; Palmer et al. 2017; Schwartz et al. 2015; Zhang et al. 2011)].

The use of large numbers of shared genes for phylogenetic inference, referred to here as phylogenomics (Daubin \& Gouy 2001; Daubin et al. 2002; Eisen \& Fraser 2003; Kumar et al. 2011), have been argued to be the most reliable option for recovering a species topology reflective of vertical descent (Andam \& Gogarten 2011; Coenye et al. 2005; Daubin et al. 2002; Galtier \& Daubin 2008). This is because the massive numbers of characters sampled is thought to dilute phylogenetic conflict within the dataset, to levels where a single robust evolutionary hypothesis is obtainable (Andam \& Gogarten 2011; Coenye et al. 2005; Cohan 2001; Daubin et al. 2002; Galtier \& Daubin 2008; Klenk \& Göker 2010). It has been suggested, particularly in bacteria, that an overall genomic core (the set of genes shared by all members of a group) exists between closely related taxa that remains evolutionarily cohesive (Coenye et al. 2005; Daubin et 
68 al. 2002). The signal found within these core genes would thus be the signal for inheritance and

69 would be appropriate for inferring the ancestral relationships (Daubin et al. 2002).

70 Despite some evidence for a genomic core (Callister et al. 2008; Daubin et al. 2002; Grote et al.

71 2012; Sarkar \& Guttman 2004), numerous studies have shown that the evolutionary trajectory of

72 genes within this subgenomic compartment may be incongruent (Bapteste et al. 2009; Dagan \&

73 Martin 2006; Jeffroy et al. 2006; Rokas et al. 2003; Thiergart et al. 2014). Dagan and Martin

74 (2006) captured this conflict in their "tree of one percent" concept. They referred to research by

75 Cicarelli and colleagues (2006), who used 31 protein sequences to recover a robust phylogenetic

76 hypothesis across a diverse set of bacterial taxa. This was after the removal of sequences

77 harbouring phylogenetic conflict from a conservative average bacterial genome of 3,000 genes.

78 In other words, the resulting phylogenetic tree that was interpreted as the evolutionary history of

79 the taxa, was based on roughly $1 \%$ of the average genome of these taxa (Dagan \& Martin 2006).

80 Additionally, research has shown that species trees may in some cases be driven by only a

81 handful of genes, particularly where contradictory species relationships are routinely observed

82 from single gene trees (Salichos \& Rokas 2013; Shen et al. 2017; Thiergart et al. 2014). It is thus

83 still unclear whether employing genome data in a concatenation-based approach is truly an

84 appropriate way of inferring evolutionary relationships, despite the popularity of this approach.

85 The incongruence often observed between gene and species trees can be attributed to two main

86 factors: phylogenetic errors and organism-level evolutionary processes (Doyle 1992; Wendel \&

87 Doyle 1998). Phylogenetic errors (i.e. stochastic errors due to the use of too little information

88 and systematic errors caused by non-phylogenetic signal) during tree inferences are mainly

89 overcome by increased character and taxon sampling (Hedtke et al. 2006; Jeffroy et al. 2006;

90 Palmer et al. 2017; Philippe et al. 2011; Pollock et al. 2002; Yokono et al. 2018). Organism-level 
91 evolutionary processes can be difficult to account for if they result in different evolutionary

92 histories for genes that cannot be integrated into a single bifurcating evolutionary hypothesis

93 (Wendel \& Doyle 1998). When phylogenetic error is excluded, incomplete lineage sorting (ILS)

94 and horizontal gene transfer (HGT) are frequently the primary organism-level processes

95 responsible for phylogenetic incongruence (Galtier \& Daubin 2008; Mallet et al. 2016; Retchless

96 \& Lawrence 2010). An ongoing debate in the scientific community is whether to concatenate and

97 risk a well-supported but incorrect species tree that also captures all phylogenetic conflict in a

98 dataset, or pool the phylogenetic signal from hundreds of gene trees in supertree or reconciliation

99 approaches (Daubin \& Gouy 2001; Galtier \& Daubin 2008; Ren et al. 2009; Retchless \&

100 Lawrence 2010; Sanderson \& Driskell 2003; Szöllősi et al. 2012; Williams et al. 2017).

101 Reconciliation approaches efficiently account for HGT because genome evolution is modelled

102 and the data produced are used for quantifying gene transfer and for inferring species trees that

103 accommodate this process (Szöllösi et al. 2012).

104 For this study, the bacterial genus Pantoea was used as a model to explore the impact of

105 potentially conflicting signal caused by organism-level evolutionary processes on the current

106 phylogenetic hypothesis for the group. This phylogeny was constructed previously using a

107 concatenation-based approach that accounted for the majority of known phylogenetic errors

108 through Maximum Likelihood analyses of partitioned datasets with appropriate evolutionary

109 models (Palmer et al. 2017). Pantoea forms part of the family Erwiniaceae in the order

110 Enterobacterales (Adeolu et al. 2016) and is closely related to the genera Erwinia and Tatumella

111 (Adeolu et al. 2016; Brady et al. 2010b; Glaeser \& Kämpfer 2015; Palmer et al. 2017). This

112 genus has been extensively studied and represents a diverse assemblage of organisms that

113 employs an array of different and important lifestyles (Brady et al. 2010a; Brady et al. 2009; 
114 Brady et al. 2010b; Lim et al. 2014; Ma et al. 2016; Palmer et al. 2016; Palmer et al. 2017;

115 Walterson \& Stavrinides 2015). Our three main objectives were to (i) determine whether or not

116 the dataset used to infer hypotheses (based on concatenation and a multi-species coalescent

117 approach) included phylogenetic conflict, and if so, how this conflict is distributed across the

118 genome; (ii) to determine whether the observed conflicts could be ascribed to organism-level

119 evolutionary processes, such as HGT and ILS; and (iii) to determine whether limited sets of

120 genes contain enough phylogenetic signal to overshadow potential conflict within the dataset in

121 order to obtain phylogenies resembling the species phylogenetic hypotheses for Pantoea. To

122 achieve these objectives we investigated conflict at the level of gene partitions and at specific

123 nucleotide sites to detect recombination between the different lineages of the Pantoea species

124 phylogeny and also to compare regions that differed significantly in their nucleotide composition

125 to the rest of the alignments.

\section{Materials and Methods}

127 1. Dataset preparation

128 Shared genes for the 27 taxa of interest (Table 1) were determined with the Efficient Database

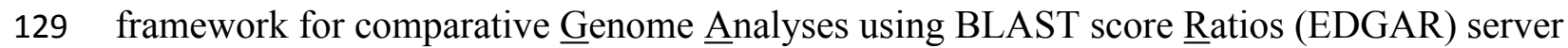

130 (Blom et al. 2016). The nucleotide sequences for all shared genes were downloaded from the

131 EDGAR server. Subsequently, the combined file of all sequences were split into individual gene

132 files. Multiple sequence alignments of genes were generated with MUSCLE (Edgar 2004) as part

133 of CLC Main Workbench v 7.6 (CLC Bio). This was followed by manual inspection and

134 correction of alignments in BioEdit v. 7.0.9 (Hall 2011) to ensure that the correct reading-frame

135 was selected for all genes. Genes were then trimmed in BioEdit to eliminate gene length

136 variation due to potential differences in gene prediction across the different genomes. To 
137 generate concatenated datasets, the respective nucleotide and protein sequences were combined

138 with FASconCAT-G v. 1.02 (Kück \& Longo 2014).

139 2. Phylogenetic analyses

140 Approximate maximum likelihood (AML; Price et al., 2010) analyses were performed on all

141 individual protein sequences, as well as, on the concatenated protein and nucleotide sequence

142 data matrices. For individual gene trees, analyses were performed in a sequential manner,

143 utilising an in-house python script (File S1). For computational efficiency, AML analyses were

144 employed in this study instead of traditional maximum likelihood (ML) analyses in alternate

145 software. Time estimates for the construction of a single gene tree based on ML is ca. 27

146 minutes/gene [RAxML v. 8.0.20 (Stamatakis 2014)] versus ca. 4 minutes/gene for AML

147 (FastTree v. 2.1), which is not surprising as up to 100 times speed increases were reported

148 previously (Price et al. 2010). All AML phylogenies were constructed with FastTree v. 2.1 (Price

149 et al. 2010) using default settings. When the relationships obtained from concatenated AML

150 analyses were not robustly supported ( $\mathrm{SH}$-support $>0.95$ ), these relationships were verified using

151 RAxML v. 8.0.20 (Stamatakis 2014).

152 A multi-species coalescent (MSC) approach (Mirarab et al. 2014) was employed to construct a

153 species tree from the individual gene phylogenies. This summary method was used to reconstruct

154 a species tree, in the presence of potential ILS (Mirarab et al. 2014), by subjecting the unrooted

155 AML phylogenies to an MSC analysis in ASTRAL v. 5.6.3 (Mirarab et al. 2014). Outputs were

156 indicated with branch lengths in coalescent units and support values for the four clusters around

157 a specific branch [quartet score; (Sayyari \& Mirarab 2016)]. Additionally, three other approaches

158 were used to infer the Pantoea species tree. The first involved inference of a Neighbour-Joining

159 (NJ) tree using distances based on Average Nucleotide Identity [ANI; (Richter \& Rosselló-Móra 
160 2009)] values. These were available from a previous study (Palmer et al. 2017) and used to 161 generate a pairwise distance matrix in Microsoft Excel ${ }^{\mathrm{TM}}$ from which a NJ tree was inferred 162 using MEGA v. 6.06 (Tamura et al. 2013). Note that this precluded resampling of the data for 163 evaluating branch support. Secondly, a Neighbor-Net network was inferred from the concatenated nucleotide data using default settings in SplitsTree v. 4 (Huson \& Bryant 2005). Thirdly, this software was also used to construct a consensus network from the single gene AML phylogenies with a zero threshold (exclude no trees) and edge weights set to count.

To determine the degree of congruence and distribution of signal across individual gene genealogies relative to the Pantoea species phylogenies, individual phylograms were manually inspected. During this process, gene genealogies supporting specific backbone nodes (that were consistently recovered using multiple inference approaches) within the Pantoea species phylogenies were identified. This was done by evaluating a set of twelve query hypotheses

172 (representing all of the internal backbone nodes in the Pantoea species trees) against each of the individual gene genealogies to determine whether they contained and/or supported the expected

174 nodes. Each genealogy was then marked as 1) fully supporting, 2) supporting, but with other taxa nested, 3) not supporting or 4) lacking signal for the specific node depicted in the query hypothesis. The signal obtained from each of the different gene trees were then related back to

177 the physical order of the shared genes as they appear in the genome of P. agglomerans (Lim et al. 2014), to determine whether specific signal patterns could be associated to areas of the genome.

As an indication of how phylogenetic conflicts were distributed across the concatenated nucleotide alignment, incongruent signals for the $P$. dispersa and $P$. ananatis lineages were investigated. For these purposes, the nucleotide sites causing incongruence in the Neighbor-Net 
183 network was noted and related back to the gene identifier. These data were visualised across the

184 concatenated alignment using Circos v. 0.69 (Krzywinski et al. 2009).

185 3. Recombination detection

186 To determine whether recombination, as an organism-level evolutionary process, could have

187 contributed to phylogenetic conflict within the dataset, genes with possible signals for

188 recombination were identified. This was done by subjecting the concatenated data matrix to the

189 Recombination Detection Program (RDP) v. 4.84 (Martin et al. 2015), to test for recombination

190 breakpoints using five genetic distance-based methods (RDP, GENECONV, MaxChi, Chimaera

191 and 3Seq). RDP employs a sliding window to calculate pairwise distances between all unique

192 taxon triplets for parsimony informative sites. Regions in contradiction to a UPGMA

193 dendrogram, constructed from all sites, are identified as potentially recombinant (Martin \&

194 Rybicki 2000). The GENECONV method entails pairwise comparisons of all polymorphic sites

195 within the alignment to identify higher than expected similarity over unusually long regions

196 compared to the rest of the alignment (Padidam et al. 1999). MaxChi identifies potential

197 recombination breakpoints by examining differences in the proportions of variable polymorphic

198 sites using a sliding window to calculate pairwise $\chi^{2}$ values (Smith 1992). The Chimaera

199 approach is in essence a modification of the MaxChi method, where triplets are screened using a

200 sliding window for only polymorphic sites where recombinants match one of the parental

201 sequences (Posada \& Crandall 2001). Lastly, the 3Seq method uses the same character set as

202 Chimaera to query each sequence within each triplet combination to determine if it could be a

203 possible recombinant of the other two sequences (Boni et al. 2007). These data were also plotted

204 on the concatenated alignment using Circos v. 0.69 (Krzywinski et al. 2009).

205 4. Randomised subset phylogenetic analyses 
206 To determine whether limited sets of genes contained sufficient phylogenetic signal to overcome 207 phylogenetic conflict within the dataset, randomised subsets of $20,50,60,70,80,90,100,110$ 208 and 120 genes were constructed. For this purpose, genes were randomly identified in Microsoft

209 Excel $^{\mathrm{TM}}[=$ RANDBETWEEN $(1,1357)]$ without resampling. The concatenation and phylogenetic 210 analyses were conducted in the same manner as described above. In all cases, ten individual data 211 subsets were constructed, followed by obtaining a strict and majority rule consensus tree of the 212 ten phylograms of each gene set (i.e., 20, 50, 60, 70, 80, 90, 100, 110 or 120 genes).

\section{Results}

\section{Detecting phylogenetic conflict}

215 Using the AML approach, a robust and well-supported evolutionary hypothesis regarding the

216 species relationships in Pantoea was obtained. The AML phylogeny was based on 337,780

217 amino acid columns corresponding to the protein sequences of 1,357 genes (Fig. 1). This

218 phylogeny was also congruent with the phylogeny obtained with a larger taxon set for Pantoea,

219 Erwinia, Tatumella and outgroup taxa by Palmer et al. (2017), where ML inferences were performed with the appropriate evolutionary models for each gene partition. The only exceptions

221 were the sister-grouping between $P$. agglomerans and $P$. vagans in the current tree, however due 222 to their close relatedness this is not an uncommon problem, and the grouping of $P$. deleyi and $P$. 223 anthophila. Similarly, a robust and equally well-supported phylogeny was obtained using the 224 MSC approach where the species tree was inferred from the set of 1,357 individual gene trees 225 (Fig. 1 and S1). Overall, the MSC topology was also congruent with the phylogeny obtained by 226 Palmer et al., (2017). Exceptions were only observed at nodes at tips or leave nodes (i.e. the 227 groupings observed in the $P$. agglomerans and $P$. dispersa lineages). 
228 The AML and MSC topologies were highly congruent (Fig. 1). The only differences were those

229 regarding relationships within the $P$. dispersa lineage and the $P$. agglomerans lineage (both

230 topologies also lacked support for the relationships within this lineage). In terms of the MSC

231 topology, comparison of the quartet scores for the main, the first alternative and second

232 alternative topologies possible at each node (Sayyari \& Mirarab 2016), showed that the nodes

233 where quartet scores between the topologies differed very little (where quartet scores for the

234 three alternatives were almost equal) were generally those responsible for incongruence between

235 the topologies inferred using different approaches (Fig. 1 and S1; particularly within the $P$.

236 agglomerans and $P$. dispersa lineages). This suggests that none of these approaches are

237 particularly robust when resolving closely related or undersampled lineages close to the leaves of 238 the phylogenies.

239 The network approaches indicated a large amount of conflicting signal within the data. This was

240 evident in the Neighbor-Net network (Fig. 2 and File S2) based on the concatenated nucleotide

241 data matrix (1,010,946 bases), as well as the Consensus Network (Fig. S2 and File S3) of the

242 individual gene trees (1,357 protein sequences). These conflicting signals were particularly

243 prevalent at the deeper edges of the evolutionary hypotheses, e.g. the $P$. dispersa lineage

244 compared to the $P$. ananatis lineage (denoted A and B in Fig. 1). However, despite the presence

245 of this conflict, the evolutionary hypotheses obtained with the networks, overall, reflected the

246 relationships obtained for the AML and MSC phylogenies (Fig. 1, 2 and S2). Furthermore, the

247 topology obtained for the ANI-based distances was mostly congruent to the lineages recovered

248 from the various species tree inference approaches (Fig. S3). All backbone nodes that were

249 consistently recovered in the other approaches, were also recovered with the ANI-based

250 distances with the exception of $P$. eucrina grouping as sister to the singleton, $P$. septica. 
251 To determine the degree of incongruence caused by phylogenetic conflict, comparisons of all

252 individual gene trees were evaluated against a set of twelve query phylogenetic hypotheses (Fig.

253 3A). These query hypotheses were constructed to evaluate monophyly of lineages or groups

254 across the backbone of the Pantoea species phylogenies, thus shallower nodes near the tips of the

255 trees (leaves) were not considered. None of the 1,357 gene trees were fully congruent with the

256 respective phylogenetic hypotheses of Pantoea. Of the individual gene trees, only six genes

257 supported all the nodes in the backbone for the groupings observed previously (File S4).

258 Additionally, seven gene genealogies produced polytomies of taxa and thus were marked as

259 containing no signal for any of the nodes observed in the phylogenetic hypotheses of Pantoea

260 (Fig. 4 and File S4). The remaining gene trees supported at least one of the nodes in the

261 backbone observed in the Pantoea species trees. Exclusion from the concatenated analyses of

262 either the six backbone-supporting genes or the seven genes providing no resolution among taxa,

263 still provided the same overall topology, with the exception of the grouping of P. agglomerans,

$264 P$. vagans and P. eucalypti (Fig. S4A and B) that lacked statistical support. The phylogenies

265 constructed from the concatenated datasets with only the six backbone-supporting genes and

266 only the seven genes showing no signal (confirmed with RAxML v. 8.0.20; Fig. 3H), also

267 allowed the recovery of a mostly congruent phylogeny to that of the expected topology, but with

268 very low or no support at a number of nodes and slight interspecies differences in the $P$.

269 agglomerans lineage and the position of singleton taxa (Fig. 3H, Fig. S5A and B).

270 Based on these topology comparisons, it appeared that the signal supporting different nodes

271 across the Pantoea species phylogenies were supported by different genes. As a means to

272 investigate the distribution of phylogenetic conflict at the gene partition-level across the genome,

273 the signal for each gene was plotted against the genome of P.agglomerans (Fig. 3C). All shared 
274 genes were localized to the chromosome of $P$. agglomerans. This analysis also revealed that

275 signal for all nodes were randomly distributed across the chromosome of P. agglomerans and no

276 apparent patterns of shared signal were detected for adjacent genes (Fig. 3C).

277 To interrogate the distribution of conflict across the dataset at the nucleotide-level, nucleotide

278 positions in phylogenetic conflict with relationships observed within the Pantoea species

279 phylogeny were identified. Of the 1,010,946 bases within the nucleotide alignment, 493,834

280 bases (48.7\%) were identical across all taxa, with 517,112 nucleotide positions being variable

281 between taxa. For these analyses the $P$. ananatis lineage, with the least conflicting signal within

282 the dataset (Fig. 2: edge A), and the P. dispersa lineage, with the most conflicting signal within

283 the dataset (Fig. 2: edge B), were investigated as a best and worst case scenario, respectively. For

284 the $P$. ananatis and $P$. dispersa lineages, a total of 1,764 and 3,856 nucleotide sites, respectively,

285 supported relationships differing from the Pantoea species phylogenies (Fig. 2 points A and B

286 File S5). However, these sites were distributed across the concatenated alignment and were not

287 localized to specific genomic regions (Fig. 5).

288 Recombination as a source of phylogenetic conflict

289 Using RDP, a total of 276 potential recombination events were detected (File S6), with 166 of

290 these indicated as potentially caused by evolutionary processes other than recombination (Martin

291 et al. 2015). This yielded 110 likely recombination events, occurring across 54 regions of the

292 concatenated sequence, supported by at least three different analytical methods, of which 57

293 events were supported by all five methods (File S6). However, to avoid the inclusion of

294 potentially artefactual recombination breakpoints associated with the concatenation process, we

295 only considered recombination breakpoints occurring within the boundaries of single genes. This

296 yielded a total of 15 recombination events, identified across 11 genes within the concatenated 
297 alignment (Fig. 5 and File S6). From these results, recombination break-points were detected in

298 members of all lineages within Pantoea. None of these recombination breakpoints could,

299 however, be linked to the nucleotide-level phylogenetic conflict identified (File S5).

300

301

302

303

304

305

306

307

308

309

310

311

312

313

314

315

316

317

318

\section{Phylograms from limited sets of genes}

The topology of the Pantoea species phylogenies could be recovered by some of the randomised subsets of 20, 50 and 60 genes, whereas all subsets containing the information for 70 or more genes recovered these nodes. Within each set of ten replicate data subsets, the length of individual alignments differed depending on the length of the specific genes used to construct them (Table 2 and File S7). For example, for the 20 gene subsets, the lengths of the alignments ranged from 5,560 to 7,152 amino acid columns, while the length of the alignments for the 120 gene subsets ranged from 36,614 - 42,793 amino acid columns (Table 2).

Overall, support for the backbone of the Pantoea species tree (Fig. 3A) deteriorated with a decrease in the number of genes concatenated and analysed (Table 2). When fewer genes were analysed in the replicates, various branches collapsed and branch support decreased in the strict consensus trees (Table 2, Fig. 6 and Fig. S6). Overall, strict consensus trees from the various replicates of 70 and more genes resulted in the recovery of a phylogeny congruent with the species phylogenies of Pantoea, however multiple individual replicates of the smaller datasets produced trees that were largely incongruent with these hypotheses. Only the trees from multigene subsets of 70 or more genes, consistently allowed robust and well-supported reconstruction of the expected Pantoea species trees, specifically with regards to branches in the backbone of the phylogeny (i.e. query hypotheses; Fig. 6).

\section{Discussion}


319 This study employed a novel approach to investigate phylogenetic conflict within concatenated 320 datasets. We interrogated the distribution and effect of both phylogenetic signal and conflict at

321 the gene partition and nucleotide levels. This entailed the use of various phylogenetic analyses

322 coupled with manual inspection and evaluation of individual gene trees. These data revealed the

323 effects of phylogenetic conflict and signal in concatenated datasets, which are the input typically

324 used for phylogenomic reconstruction. Our findings support the idea that all genes, even if they

325 appear to be phylogenetically uninformative when analysed alone, contribute signal toward a

326 phylogenomic evolutionary hypothesis and that the obtained topology is not driven by single

327 genes. This is reminiscent of Aristotle's idea of synergism that "the whole is greater than the sum

328 of its parts". In other words, by concatenating single genes a synergistic effect is achieved, where

329 the combined data seems to be superior to that of the proverbial sum of the signal.

330

331

332

333

334

335

336

337

338

339

340

As demonstrated previously (Palmer et al. 2017), the full set of shared genes allowed

reconstruction of a robustly supported phylogenetic hypothesis for Pantoea using AML. In fact,

it is quite common to obtain a robust, highly supported phylogeny through concatenation of all

shared genes, despite the incongruent nature of individual gene trees (Hedtke et al. 2006; Jeffroy

et al. 2006; Rokas et al. 2003; Salichos \& Rokas 2013; Thiergart et al. 2014). However, none of

our single gene genealogies was fully congruent with the phylogenomic species tree of Pantoea,

while only six genes allowed recovery of the backbone of the species phylogeny. This was not

surprising as various previous studies showed that very few or no genes typically support a

particular species phylogeny fully (Dagan \& Martin 2006; Hedtke et al. 2006; Jeffroy et al. 2006;

Rokas et al. 2003; Salichos \& Rokas 2013; Thiergart et al. 2014). In contrast to conclusions

drawn previously (Thiergart et al. 2014), most of the Pantoea gene trees supported at least some 
341 of the nodes within the species phylogeny. In other words, support for the respective nodes was

342 not necessarily obtained from the same genes but rather scattered across different genes.

343 A fully resolved, well-supported phylogeny was obtained using the MSC approach, although it

344 was not congruent with the AML tree regarding relationships at the tips of the trees.

345 Concatenation is thought to be superior to MSC-based species trees if ILS is low, while MSC

346 models perform better in the presence of moderate ILS (Mirarab et al. 2014). As our MSC and

347 AML trees correspond perfectly regarding backbone nodes, these are strong hypotheses that may

348 approach the real relationships among these taxa. However, because organism-level evolutionary

349 processes were not quantified in this study, we cannot exclude the possibility that ILS were

350 responsible for the incongruences observed. Our study therefore highlights that alternative

351 approaches that model genome evolution, quantify organism-level evolutionary processes

352 (Szöllősi et al. 2012; Williams et al. 2017), and that focusses on leave taxa are needed to fully

353 resolve the species tree of a diverse assemblage such as Pantoea. This may be particularly true

354 when taxa are very closely related or when the lineages in question are undersampled.

355 The random distribution of signal across the Pantoea genome is supported by the recovery of

356 overall congruent subset phylogenies from random sub-samplings of gene sequences. Due to this

357 random distribution, one should be able to obtain sufficient signal to reconstruct the species

358 phylogeny by randomly sampling enough genes from the genome (Dutilh et al. 2004; Gadagkar

359 et al. 2005). From our data, this idea was tested with consensus trees of 10 replicates with 20, 50,

$36060,70,80,90,100,110$ and 120 genes. We found that, with a decrease in the number of genes

361 analysed, support for the backbone and the deeper branches decreased incrementally, as has also

362 been observed previously (Rokas et al. 2003). Therefore, for these data and taxon set, it appear

363 that at least 70 randomly selected genes from the genome is required to obtain a relatively robust, 
364 well-supported phylogeny, particularly to reconstruct the deeper relationships within and among

365 the genera. Multi-gene phylogenies based on 70 genes may thus provide sufficiently robust

366 hypotheses so that complete genome sequence data may not be required, our work suggests that

367 sufficient data may be obtained from low level sequencing, although verification of this notion in

368 other taxon sets is required.

369 The species tree hypotheses for Pantoea was generally also supported by the two network

370 approaches employed here. Both accommodated non-vertical and non-phylogenetic signal

371 (introduced through systematic error) as inferred from nucleotide data, as well as the individual

372 gene trees (Bryant \& Moulton 2002; Holland \& Moulton 2003; Holland et al. 2005). These

373 methods produced networks in which the overall clustering patterns were generally congruent

374 with that obtained through gene concatenation-based phylogenomic inferences. This would not

375 have happened if insufficient signal [i.e. stochastic error (Jeffroy et al. 2006; Philippe et al. 2011;

376 Rosenberg \& Kumar 2003)] or reconstruction artefacts [i.e. systematic error (Hedtke et al. 2006;

377 Hillis 1998; Pollock et al. 2002; Zwickl \& Hillis 2002)] were responsible for the observed

378 relationships in the species trees. If conflict, particularly in the form of HGT and ILS,

379 dominated the dataset, the splits graphs would not have such high overall congruence to the

380 species trees (Bryant \& Moulton 2002), however, more in-depth future studies are required to

381 fully elucidate the role and amount of organism-level evolutionary processes in the evolution of

382 these taxa. Compared to previous analyses, often employing a limited gene set (Chen et al. 2013;

383 Kennedy et al. 2005), more box-like structures were observed in Pantoea networks, particularly

384 in deeper edges. However, these boxes were generally smaller, where the increased number of

385 boxes indicate more alternate or conflicting relationships, while the shorter edges correlate to the 386 particular relationships being observed less frequently. If one considers that these conflicts are 
387 visualized for the full shared gene set, the level of conflict appears to be relatively low and

388 comparable to that seen in other bacteria (Retchless \& Lawrence 2010), but considerably lower

389 than taxa undergoing extensive HGT (Doroghazi \& Buckley 2010). The generally low level of

390 conflict thus supports the idea that sufficient phylogenetic signal is present within the

391 concatenated dataset to overshadow the limited conflict present.

392 Overall, the relationships obtained using the ANI-based distance approach were mostly

congruent to the species trees. The incongruences that were present can be ascribed to the fact

that ANI is notoriously unreliable as an indicator of relatedness, especially among more distantly

related taxa (Konstantinidis \& Tiedje 2007; Palmer et al. 2017; Qin et al. 2014; Rosselló-Mora

2005). This phenomenon is the reason why many prokaryotic taxonomist would rather purport

the use of Average Amino Acid Identity (AAI) values at this level (Konstantinidis \& Tiedje

2007; Qin et al. 2014; Rosselló-Mora 2005), as substitution saturation and other factors resulting

from endogenous evolutionary processes may be responsible for the decline in informativeness

of this metric, the more distantly related the taxa become (Palmer et al. 2017).

401

402

403

404

405

406

407

408

409

We investigated the phylogenetic conflict within the Pantoea dataset for the two lineages in which we observed the least and most phylogenetic conflict. Respectively, these were the $P$. ananatis lineage, which includes plant pathogenic species, and the $P$. dispersa lineage, which includes generalists (Palmer et al. 2018; Palmer et al. 2017; Walterson \& Stavrinides 2015). The number of nucleotide sites supporting alternate topologies to the species trees were limited, with only $0.75 \%$ of variable nucleotide sites $(3,856$ sites out of 517,112$)$ supporting conflicting topologies in the $P$. dispersa lineage. Also, the remaining variable sites did not necessarily support the species relationships observed in the species trees, because different genes and nucleotide positions supported different nodes within the species phylogenies. Moreover, the 
410 conflicting signal within the dataset was not localised to specific genomic regions or genes, but

411 rather, was randomly distributed. Taken together, these results thus suggest that i) the use of

412 network approaches for constructing phylogenies can be extremely valuable for identifying

413 phylogenetic conflicts in datasets (Bryant \& Moulton 2002; Holland \& Moulton 2003; Holland

414 et al. 2005), and ii) organism-level evolutionary processes like HGT and/or ILS impacts different

415 lineages and taxa to varying degrees.

416 Conflicting phylogenetic signal in the Pantoea dataset could potentially result from

417 recombination events that led to gene conversions between species (Daubin et al. 2003; Fraser et

418 al. 2007; Holmes et al. 1999; Posada \& Crandall 2001; Posada \& Crandall 2002). We found

419 evidence for at least 15 recombination events in 11 shared genes in the dataset. We attributed

420 these to recent instances of recombination, because older organism-level evolutionary events,

421 particularly ancient HGT and ILS (Knowles 2009; Meng \& Kubatko 2009; Retchless \&

422 Lawrence 2010), become difficult to detect due to deterioration of signals by endogenous

423 evolutionary processes (Daubin et al. 2003). It is also difficult to distinguish between these

424 organism-level evolutionary processes as their signals may appear very similar (Knowles 2009;

425 Nosil 2008; Wendel \& Doyle 1998) and future studies would be required to tease apart the roles

426 of these processes in Pantoea. Nevertheless, identification of some of these organism-level

427 evolutionary events in Pantoea provides possible mechanisms for how phylogenetic conflict

428 could have been introduced into the data.

429 The use of all shared genomic information allowed for the recovery of robustly supported

430 relationships, overcoming the weaknesses observed in individual gene datasets (Andam \&

431 Gogarten 2011; Daubin et al. 2002; Gadagkar et al. 2005; Galtier \& Daubin 2008). In contrast to

432 a previous similar study by Thiergart et al. (2014), comparison of the single gene trees were 
433 specifically performed with backbone nodes (excluding taxa closer to the tips of the trees) with

434 the aid of the query hypotheses, which allowed us to interrogate each node and its associated

435 signal manually. Although Thiergart et al. (2014) also compared nodes between concatenated

436 trees and the single gene phylogenies, their comparisons were focussed only on the recovery of

437 identical nodes, which likely overestimated the effect of finer differences between the trees,

438 leading them to their conclusion that the signal associated with the backbone or deeper nodes of

439 their concatenated phylogenies are not preserved in single gene trees. Based on our data, three of

440 the query hypotheses evaluated (see Fig. 3 B and S1) had a large proportion of individual gene

441 trees that did not support the expected monophyly of the taxon groups specified. These were

442 hypothesis 5 in which $P$. septica is a singleton taxon placed as sister to the $P$. agglomerans and

443 P. ananatis lineages (48.9\% trees), hypothesis 9 in which $P$. cypripedii and Pantoea sp. At-9b

444 are placed as sister to the $P$. rodasii and $P$. dispersa lineages $(40.4 \%$ trees $)$ and hypothesis 10 in

445 which the $P$. rodasii and $P$. dispersa lineages are placed as sister groups $(35.2 \%$ trees $)$. In these

446 instances, limited species have been sampled for the respective lineages. This undersampling of

447 the diversity may contribute to the lack in robust recovery of the lineages due to large systematic

448 error in the smaller datasets. In future, increased taxon sampling may resolve these problematic

449 relationships more accurately in smaller datasets like those employed for the single gene trees

450 (Hedtke et al. 2006; Pollock et al. 2002).

451 Comparison of single gene trees with phylogenies obtained from concatenated datasets, presents

452 both a philosophical and logical quandary. It is widely accepted that single gene phylogenies,

453 often with very limited or no statistical support, cannot be equated to a species phylogeny

454 (Degnan \& Rosenberg 2006; Doyle 1992; Maddison 1997; Pamilo \& Nei 1988; Rosenberg

455 2002). Despite the common practice of evaluating the robustness of a species phylogeny 
456 constructed from thousands or millions of characters, by its topological congruence to single

457 gene trees (Bapteste et al. 2009; Ciccarelli et al. 2006; Dagan \& Martin 2006), these phylogenies

458 are clearly not directly comparable and no conclusions regarding species evolution should be

459 drawn from raw tree topology comparisons. This rationale is like comparing single molecules

460 with chemical compounds and being disappointed that they do not share the same characteristics.

461 Based on our data, the signal required for reconstructing a species phylogeny is dispersed and the

462 only appropriate comparison of single gene trees to species trees would be when focus is placed

463 on the evolution of a particular gene or when species trees are inferred from single gene trees, as

464 with the MSCM analyses.

465 Our findings confirm the robustness of phylogenies constructed from genomic data, based on the 466 synergistic effect of combined genes, despite high levels of incongruence between individual

467 gene trees. This is due to the phylogenetic signal for different nodes within the species

468 phylogeny being distributed across the genome at higher levels than the randomly distributed

469 conflicts within the dataset. These findings support previous conclusions suggested by several

470 authors (Andam \& Gogarten 2011; Daubin et al. 2002; Galtier \& Daubin 2008; Retchless \&

471 Lawrence 2010) (Rokas 2003), based on comparisons of single gene phylogenies with super

472 trees and concatenated analyses using tree-to-tree distance approaches (Daubin et al. 2002;

473 Retchless \& Lawrence 2010). Our results also suggest that the robustness of evolutionary

474 hypotheses from whole genome data should be evaluated with phylogenetic network approaches

475 that can depict conflicts, due to evolutionary processes or phylogenetic error, within the dataset

476 (Bryant \& Moulton 2002; Holland \& Moulton 2003; Huson \& Bryant 2005). By employing such

477 a total-evidence based approach, one would be able to recover a more realistic evolutionary

478 hypothesis, particularly in terms of the deeper relationships, that also serves as an initial 
479 indication of the impact of organism-level evolutionary processes. Ultimately, such detailed 480 evolutionary analyses would be invaluable for understanding the speciation process and for 481 studying the development and distribution of important biological characteristics. Furthermore, 482 our data also suggests that alternative approaches, focussing specifically on organism-level evolutionary processes, possibly at the population level, may be required to resolve relationships and elucidate the evolutionary history of younger taxa or leaves, where these processes may be rampant and phylogenetic incongruence highly prevalent.

\section{Conclusions}

487 We found that phylogenetic conflict, potentially caused by organism-level evolutionary

488 processes, was present in our phylogenomic dataset at both the gene partition and nucleotide levels. Although this non-phylogenetic signal could result from organism-level evolutionary

490 process, like HGT and ILS, more in-depth analyses is needed to differentiate between these 491 processes and to quantify the overall impact of these processes on the evolutionary history of the 492 taxa. From our results it appeared that both signal and noise are randomly distributed across the 493 genome and that all genes included in a concatenation-based phylogenomic analysis contribute signal toward the possible species tree. In other words, for Pantoea at least, phylogenies

495 constructed from concatenated datasets are not driven by single genes, but rather that the signal 496 from individual genes work synergistically to provide robust phylogenies.

\section{Acknowledgements}

We would like to sincerely thank the reviewers, Dr. David Waite, Dr. Luis M. Rodriguez-R and an anonymous reviewer for valuable suggestions and significant contributions toward improving 500 the manuscript. 
501

502

503

504

505

506

507

508

509

510

511

512

513

514

515

516

517

518

519

520

521

522

523

524

525

526

527

528

529

530

531

532

533

534

535

536

537

538

539

540

541

542

543

544

545

\section{References}

Abdul Rahman N, Parks DH, Vanwonterghem I, Morrison M, Tyson GW, and Hugenholtz P. 2016. A Phylogenomic Analysis of the Bacterial Phylum Fibrobacteres. Frontiers in Microbiology 6. 10.3389/fmicb.2015.01469

Adeolu M, Alnajar S, Naushad S, and S. Gupta R. 2016. Genome-based phylogeny and taxonomy of the 'Enterobacteriales': proposal for Enterobacterales ord. nov. divided into the families Enterobacteriaceae, Erwiniaceae fam. nov., Pectobacteriaceae fam. nov., Yersiniaceae fam. nov., Hafniaceae fam. nov., Morganellaceae fam. nov., and Budviciaceae fam. nov. International Journal of Systematic and Evolutionary Microbiology 66:5575-5599. doi:10.1099/ijsem.0.001485

Andam CP, and Gogarten JP. 2011. Biased gene transfer in microbial evolution. Nature reviews Microbiology 9:543.

Bapteste E, O'Malley MA, Beiko RG, Ereshefsky M, Gogarten JP, Franklin-Hall L, Lapointe F-J, Dupré J, Dagan T, Boucher Y, and Martin W. 2009. Prokaryotic evolution and the tree of life are two different things. Biology Direct 4:34. 10.1186/1745-6150-4-34

Beukes CW, Palmer M, Manyaka P, Chan WY, Avontuur JR, van Zyl E, Huntemann M, Clum A, Pillay M, and Palaniappan K. 2017. Genome data provides high support for generic boundaries in Burkholderia sensu lato. Frontiers in Microbiology 8:1154.

Blom J, Kreis J, Spänig S, Juhre T, Bertelli C, Ernst C, and Goesmann A. 2016. EDGAR 2.0: an enhanced software platform for comparative gene content analyses. Nucleic acids research 44:W22-W28.

Boni MF, Posada D, and Feldman MW. 2007. An exact nonparametric method for inferring mosaic structure in sequence triplets. Genetics 176:1035-1047.

Brady CL, Cleenwerck I, Venter SN, Engelbeen K, De Vos P, and Coutinho TA. 2010a. Emended description of the genus Pantoea, description of four species from human clinical samples, Pantoea septica sp. nov., Pantoea eucrina sp. nov., Pantoea brenneri sp. nov. and Pantoea conspicua sp. nov., and transfer of Pectobacterium cypripedii (Hori 1911) Brenner et al. 1973 emend. Hauben et al. 1998 to the genus as Pantoea cypripedii comb. nov. International Journal of Systematic and Evolutionary Microbiology 60:2430-2440.

Brady CL, Venter SN, Cleenwerck I, Engelbeen K, Vancanneyt M, Swings J, and Coutinho TA. 2009. Pantoea vagans sp. nov., Pantoea eucalypti sp. nov., Pantoea deleyi sp. nov. and Pantoea anthophila sp. nov. International Journal of Systematic and Evolutionary Microbiology 59:2339-2345.

Brady CL, Venter SN, Cleenwerck I, Vandemeulebroecke K, De Vos P, and Coutinho TA. 2010b. Transfer of Pantoea citrea, Pantoea punctata and Pantoea terrea to the genus Tatumella emend. as Tatumella citrea comb. nov., Tatumella punctata comb. nov. and Tatumella terrea comb. nov. and description of Tatumella morbirosei sp. nov. International Journal of Systematic and Evolutionary Microbiology 60:484-494.

Bryant D, and Moulton V. 2002. NeighborNet: An agglomerative method for the construction of planar phylogenetic networks. WABI: Springer. p 375-391.

Callister SJ, McCue LA, Turse JE, Monroe ME, Auberry KJ, Smith RD, Adkins JN, and Lipton MS. 2008. Comparative Bacterial Proteomics: Analysis of the Core Genome Concept. PLOS ONE 3:e1542. 10.1371/journal.pone.0001542

Chen S, Kim D-K, Chase MW, and Kim J-H. 2013. Networks in a Large-Scale Phylogenetic Analysis: Reconstructing Evolutionary History of Asparagales (Lilianae) Based on Four Plastid Genes. PLOS ONE 8:e59472. 10.1371/journal.pone.0059472

Ciccarelli FD, Doerks T, Von Mering C, Creevey CJ, Snel B, and Bork P. 2006. Toward automatic reconstruction of a highly resolved tree of life. Science 311:1283-1287. 
546

Coenye T, Gevers D, de Peer YV, Vandamme P, and Swings J. 2005. Towards a prokaryotic genomic taxonomy. FEMS microbiology reviews 29:147-167.

Cohan FM. 2001. Bacterial species and speciation. Systematic Biology 50:513-524.

Dagan T, and Martin W. 2006. The tree of one percent. Genome biology 7:118. 10.1186/gb-2006-7-10118

Daubin V, and Gouy M. 2001. Bacterial molecular phylogeny using supertree approach. Genome Informatics 12:155-164.

Daubin V, Gouy M, and Perriere G. 2002. A phylogenomic approach to bacterial phylogeny: evidence of a core of genes sharing a common history. Genome Research 12:1080-1090.

Daubin V, Moran NA, and Ochman H. 2003. Phylogenetics and the Cohesion of Bacterial Genomes. Science 301:829-832. 10.1126/science.1086568

Degnan JH, and Rosenberg NA. 2006. Discordance of Species Trees with Their Most Likely Gene Trees. PLOS Genetics 2:e68. 10.1371/journal.pgen.0020068

Doroghazi JR, and Buckley DH. 2010. Widespread homologous recombination within and between Streptomyces species. The Isme Journal 4:1136. 10.1038/ismej.2010.45

https://www.nature.com/articles/ismej201045\#supplementary-information

Doyle JJ. 1992. Gene trees and species trees: molecular systematics as one-character taxonomy. Systematic Botany:144-163.

Dutilh BE, Huynen MA, Bruno WJ, and Snel B. 2004. The consistent phylogenetic signal in genome trees revealed by reducing the impact of noise. Journal of molecular evolution 58:527-539.

Edgar RC. 2004. MUSCLE: multiple sequence alignment with high accuracy and high throughput. Nucleic acids research 32:1792-1797.

Eisen JA, and Fraser CM. 2003. Phylogenomics: intersection of evolution and genomics. Science 300:1706.

Fraser C, Hanage WP, and Spratt BG. 2007. Recombination and the nature of bacterial speciation. Science 315:476-480.

Gadagkar SR, Rosenberg MS, and Kumar S. 2005. Inferring species phylogenies from multiple genes: concatenated sequence tree versus consensus gene tree. Journal of Experimental Zoology Part B: Molecular and Developmental Evolution 304:64-74.

Galtier N, and Daubin V. 2008. Dealing with incongruence in phylogenomic analyses. Philosophical Transactions of the Royal Society of London B: Biological Sciences 363:4023-4029.

Glaeser SP, and Kämpfer P. 2015. Multilocus sequence analysis (MLSA) in prokaryotic taxonomy. Systematic and applied microbiology 38:237-245.

Grote J, Thrash JC, Huggett MJ, Landry ZC, Carini P, Giovannoni SJ, and Rappé MS. 2012. Streamlining and Core Genome Conservation among Highly Divergent Members of the SAR11 Clade. mBio 3. 10.1128/mBio.00252-12

Gupta RS, Naushad S, and Baker S. 2015. Phylogenomic analyses and molecular signatures for the class Halobacteria and its two major clades: a proposal for division of the class Halobacteria into an emended order Halobacteriales and two new orders, Haloferacales ord. nov. and Natrialbales ord. nov., containing the novel families Haloferacaceae fam. nov. and Natrialbaceae fam. nov. International Journal of Systematic and Evolutionary Microbiology 65:1050-1069. doi:10.1099/ijs.0.070136-0

Hall T. 2011. BioEdit: an important software for molecular biology. GERF Bull Biosci 2:60-61.

Hedtke SM, Townsend TM, and Hillis DM. 2006. Resolution of phylogenetic conflict in large data sets by increased taxon sampling. Systematic biology 55:522-529.

Hillis DM. 1998. Taxonomic sampling, phylogenetic accuracy, and investigator bias. Systematic biology 47:3-8. 
592

593

594

595

596

597

598

599

600

601

602

603

604

605

606

607

608

609

610

611

612

613

614

615

616

617

618

619

620

621

622

623

624

625

626

627

628

629

630

631

632

633

634

635

636

637

638

Holland B, and Moulton V. 2003. Consensus networks: a method for visualising incompatibilities in collections of trees. Algorithms in bioinformatics:165-176.

Holland BR, Jermiin LS, and Moulton V. 2005. Improved consensus network techniques for genome-scale phylogeny. Molecular biology and evolution 23:848-855.

Holmes EC, Urwin R, and Maiden M. 1999. The influence of recombination on the population structure and evolution of the human pathogen Neisseria meningitidis. Molecular biology and evolution 16:741-749.

Huson DH, and Bryant D. 2005. Estimating phylogenetic trees and networks using SplitsTree 4. Manuscript in preparation, software available from www splitstree org.

Jeffroy O, Brinkmann H, Delsuc F, and Philippe H. 2006. Phylogenomics: the beginning of incongruence? Trends in Genetics 22:225-231. https://doi.org/10.1016/j.tig.2006.02.003

Jolley KA, Bliss CM, Bennett JS, Bratcher HB, Brehony C, Colles FM, Wimalarathna H, Harrison OB, Sheppard SK, Cody AJ, and Maiden MCJ. 2012. Ribosomal multilocus sequence typing: universal characterization of bacteria from domain to strain. Microbiology 158:1005-1015. doi:10.1099/mic.0.055459-0

Kennedy M, Holland BR, Gray RD, and Spencer HG. 2005. Untangling Long Branches: Identifying Conflicting Phylogenetic Signals Using Spectral Analysis, Neighbor-Net, and Consensus Networks. Systematic biology 54:620-633. 10.1080/106351591007462

Klenk H-P, and Göker M. 2010. En route to a genome-based classification of Archaea and Bacteria? Systematic and applied microbiology 33:175-182.

Knowles LL. 2009. Estimating species trees: methods of phylogenetic analysis when there is incongruence across genes. Systematic biology 58:463-467.

Konstantinidis KT, and Tiedje JM. 2007. Prokaryotic taxonomy and phylogeny in the genomic era: advancements and challenges ahead. Current opinion in microbiology 10:504-509.

Krzywinski M, Schein J, Birol I, Connors J, Gascoyne R, Horsman D, Jones SJ, and Marra MA. 2009. Circos: an information aesthetic for comparative genomics. Genome Research 19:1639-1645.

Kück P, and Longo GC. 2014. FASconCAT-G: extensive functions for multiple sequence alignment preparations concerning phylogenetic studies. Frontiers in zoology 11:81.

Kumar S, Filipski AJ, Battistuzzi FU, Kosakovsky Pond SL, and Tamura K. 2011. Statistics and truth in phylogenomics. Molecular biology and evolution 29:457-472.

Lim J-A, Lee DH, Kim B-Y, and Heu S. 2014. Draft genome sequence of Pantoea agglomerans R190, a producer of antibiotics against phytopathogens and foodborne pathogens. Journal of biotechnology 188:7-8.

Ma Y, Yin Y, Rong C, Chen S, Liu Y, Wang S, and Xu F. 2016. Pantoea pleuroti sp. nov., Isolated from the Fruiting Bodies of Pleurotus eryngii. Current microbiology 72:207-212.

Maddison WP. 1997. Gene trees in species trees. Systematic biology 46:523-536.

Mallet J, Besansky N, and Hahn MW. 2016. How reticulated are species? Bioessays 38:140-149. doi:10.1002/bies.201500149

Martin D, and Rybicki E. 2000. RDP: detection of recombination amongst aligned sequences. Bioinformatics 16:562-563.

Martin DP, Murrell B, Golden M, Khoosal A, and Muhire B. 2015. RDP4: Detection and analysis of recombination patterns in virus genomes. Virus Evolution 1.

Meehan CJ, and Beiko RG. 2014. A Phylogenomic View of Ecological Specialization in the Lachnospiraceae, a Family of Digestive Tract-Associated Bacteria. Genome Biology and Evolution 6:703-713. 10.1093/gbe/evu050

Meng C, and Kubatko LS. 2009. Detecting hybrid speciation in the presence of incomplete lineage sorting using gene tree incongruence: a model. Theoretical population biology 75:35-45. 
639

640

641

642

643

644

645

646

647

648

649

650

651

652

653

654

655

656

657

658

659

660

661

662

663

664

665

666

667

668

669

670

671

672

673

674

675

676

677

678

679

680

681

682

683

684

685

Mirarab S, Reaz R, Bayzid MS, Zimmermann T, Swenson MS, and Warnow T. 2014. ASTRAL: genome-scale coalescent-based species tree estimation. Bioinformatics 30:i541-i548.

Nosil P. 2008. Speciation with gene flow could be common. Molecular Ecology 17:2103-2106.

Padidam M, Sawyer S, and Fauquet CM. 1999. Possible emergence of new geminiviruses by frequent recombination. Virology 265:218-225.

Palmer M, de Maayer P, Poulsen M, Steenkamp ET, van Zyl E, Coutinho TA, and Venter SN. 2016. Draft genome sequences of Pantoea agglomerans and Pantoea vagans isolates associated with termites. Standards in genomic sciences 11:23.

Palmer M, Steenkamp ET, Coetzee MPA, Blom J, and Venter SN. 2018. Genome-based characterization of biological processes that differentiate closely related bacteria. Frontiers in Microbiology 9:113. 10.3389/fmicb.2018.00113

Palmer M, Steenkamp ET, Coetzee MPA, Chan W-Y, van Zyl E, De Maayer P, Coutinho TA, Blom J, Smits THM, Duffy B, and Venter SN. 2017. Phylogenomic resolution of the bacterial genus Pantoea and its relationship with Erwinia and Tatumella. Antonie van Leeuwenhoek 110:1287-1309. 10.1007/s10482-017-0852-4

Pamilo P, and Nei M. 1988. Relationships between gene trees and species trees. Molecular biology and evolution 5:568-583.

Philippe H, Brinkmann H, Lavrov DV, Littlewood DTJ, Manuel M, Wörheide G, and Baurain D. 2011. Resolving difficult phylogenetic questions: why more sequences are not enough. PLoS biology 9:e1000602.

Pollock DD, ZwickI DJ, McGuire JA, and Hillis DM. 2002. Increased taxon sampling is advantageous for phylogenetic inference. Systematic biology 51:664.

Posada D, and Crandall KA. 2001. Evaluation of methods for detecting recombination from DNA sequences: computer simulations. Proceedings of the National Academy of Sciences 98:1375713762.

Posada D, and Crandall KA. 2002. The effect of recombination on the accuracy of phylogeny estimation. Journal of molecular evolution 54:396-402.

Price MN, Dehal PS, and Arkin AP. 2010. FastTree 2-approximately maximum-likelihood trees for large alignments. PLOS ONE 5:e9490.

Qin Q-L, Xie B-B, Zhang X-Y, Chen X-L, Zhou B-C, Zhou J, Oren A, and Zhang Y-Z. 2014. A proposed genus boundary for the prokaryotes based on genomic insights. Journal of Bacteriology:JB. 0168801614.

Ren F, Tanaka H, and Yang Z. 2009. A likelihood look at the supermatrix-supertree controversy. Gene 441:119-125. https://doi.org/10.1016/j.gene.2008.04.002

Retchless AC, and Lawrence JG. 2010. Phylogenetic incongruence arising from fragmented speciation in enteric bacteria. Proceedings of the National Academy of Sciences 107:11453-11458.

Richter M, and Rosselló-Móra R. 2009. Shifting the genomic gold standard for the prokaryotic species definition. Proceedings of the National Academy of Sciences 106:19126-19131.

Rokas A, Williams BL, King N, and Carroll SB. 2003. Genome-scale approaches to resolving incongruence in molecular phylogenies. Nature 425:798.

Rosenberg MS, and Kumar S. 2003. Taxon sampling, bioinformatics, and phylogenomics. Systematic biology 52:119.

Rosenberg NA. 2002. The probability of topological concordance of gene trees and species trees. Theoretical population biology 61:225-247.

Rosselló-Mora R. 2005. Updating prokaryotic taxonomy. Journal of Bacteriology 187:6255-6257.

Salichos L, and Rokas A. 2013. Inferring ancient divergences requires genes with strong phylogenetic signals. Nature 497:327. 
686

687

688

689

690

691

692

693

694

695

696

697

698

699

700

701

702

703

704

705

706

707

708

709

710

711

712

713

714

715

716

717

718

719

720

721

722
Sanderson MJ, and Driskell AC. 2003. The challenge of constructing large phylogenetic trees. Trends in Plant Science 8:374-379. https://doi.org/10.1016/S1360-1385(03)00165-1

Sarkar SF, and Guttman DS. 2004. Evolution of the Core Genome of Pseudomonas syringae, a Highly Clonal, Endemic Plant Pathogen. Applied and Environmental Microbiology 70:1999-2012. 10.1128/aem.70.4.1999-2012.2004

Sayyari E, and Mirarab S. 2016. Fast coalescent-based computation of local branch support from quartet frequencies. Molecular biology and evolution 33:1654-1668.

Schwartz AR, Potnis N, Timilsina S, Wilson M, Patané J, Martins J, Minsavage GV, Dahlbeck D, Akhunova A, Almeida N, Vallad GE, Barak JD, White FF, Miller SA, Ritchie D, Goss E, Bart RS, Setubal JC, Jones JB, and Staskawicz BJ. 2015. Phylogenomics of Xanthomonas field strains infecting pepper and tomato reveals diversity in effector repertoires and identifies determinants of host specificity. Frontiers in Microbiology 6:535. 10.3389/fmicb.2015.00535

Shen X-X, Hittinger CT, and Rokas A. 2017. Contentious relationships in phylogenomic studies can be driven by a handful of genes. Nature Ecology \& Evolution 1:0126.

Smith JM. 1992. Analyzing the mosaic structure of genes. Journal of molecular evolution 34:126-129.

Stamatakis A. 2014. RAxML version 8: a tool for phylogenetic analysis and post-analysis of large phylogenies. Bioinformatics 30:1312-1313. 10.1093/bioinformatics/btu033

Szöllősi GJ, Boussau B, Abby SS, Tannier E, and Daubin V. 2012. Phylogenetic modeling of lateral gene transfer reconstructs the pattern and relative timing of speciations. Proceedings of the National Academy of Sciences:201202997.

Tamura K, Stecher G, Peterson D, Filipski A, and Kumar S. 2013. MEGA6: molecular evolutionary genetics analysis version 6.0. Molecular biology and evolution 30:2725-2729.

Thiergart T, Landan G, and Martin WF. 2014. Concatenated alignments and the case of the disappearing tree. BMC Evolutionary Biology 14:266.

Walterson AM, and Stavrinides J. 2015. Pantoea: insights into a highly versatile and diverse genus within the Enterobacteriaceae. FEMS microbiology reviews 39:968-984.

Wendel JF, and Doyle JJ. 1998. Phylogenetic incongruence: window into genome history and molecular evolution. Molecular systematics of plants II: Springer, 265-296.

Williams TA, Szöllősi GJ, Spang A, Foster PG, Heaps SE, Boussau B, Ettema TJ, and Embley TM. 2017. Integrative modeling of gene and genome evolution roots the archaeal tree of life. Proceedings of the National Academy of Sciences:201618463.

Yokono M, Satoh S, and Tanaka A. 2018. Comparative analyses of whole-genome protein sequences from multiple organisms. Scientific Reports 8:6800. 10.1038/s41598-018-25090-8

Zhang Z-G, Ye Z-Q, Yu L, and Shi P. 2011. Phylogenomic reconstruction of lactic acid bacteria: an update. BMC Evolutionary Biology 11:1. 10.1186/1471-2148-11-1

Zwickl DJ, and Hillis DM. 2002. Increased taxon sampling greatly reduces phylogenetic error. Systematic biology 51:588-598. 


\section{Figure Legends}

724 Figure 1. A comparison between the two species trees based on the approaches employed in this

725 study. Blue branches indicate differences in topology and branches with support of lower than

7260.95 are indicated with an asterisk. A. The approximate maximum likelihood (AML) phylogeny

727 constructed from the concatenated data matrix of the protein sequences of 1,357 genes,

728 consisting of 337,780 amino acid columns. The phylogeny was constructed with FastTree v. 2.1

729 (Price et al., 2010) with the JTT (Jones et al., 1992) evolutionary model with CAT

730 approximation. Simodaira-Hasegawa branch support values from 1,000 replicates were used. B.

731 A species phylogeny using the multispecies coalescent model as implemented in ASTRAL

732 v.5.6.3 (Mirarab et al., 2014) based on the individual phylogenies constructed from the protein

733 sequences of 1,357 genes. The scale bar indicates one coalescent unit (Mirarab et al., 2014).

734 Terminal branches are indicated as one coalescent unit, as branch lengths for taxa corresponding

735 to species can only be calculated where multiple individuals per species are analysed. Shorter

736 branches correspond to higher levels of incongruence and are generally associated with high

737 levels of incomplete lineage sorting (ILS). Support values are determined based on Bayesian

738 posterior probability values computed from the single gene tree quartet frequencies (Sayyari et

739 al., 2016).

740 Figure 2. A Neighbor-Net network constructed from p-distances with equal angles for the

741 concatenated nucleotide dataset. Overall, the configuration of the network is congruent with the

742 existing species phylogeny for the genus Pantoea. Clear separation between the P. agglomerans

743 and $P$. ananatis lineages were obtained and were also clearly distinct form the $P$. rodasii and $P$.

744 dispersa lineages. Point A denotes where signal in conflict to the grouping of the P. ananatis 
745 lineage was determined, while point B denotes where signal in conflict to the grouping of the $P$.

746 dispersa lineage was determined (see text for details).

747 Figure 3. An overview of the individual gene tree comparisons. A. The phylogenetic hypotheses

748 evaluated during topology comparisons. Each number represents a specific hypothesis, where the

749 monophyly of the group at each node was evaluated. An example is hypothesis 3 , where the

750 overall monophyly of Pantoea was evaluated. B. A relative frequency histogram depicting the

751 proportion of individual gene trees that support the phylogenetic hypotheses evaluated. Dark

752 blue indicates genes that fully supported the monophyly of the corresponding hypothesis, while

753 light blue indicates support for the monophyly of the hypothesis with additional taxa nested

754 within the group. Red indicates gene trees that were incongruent with the corresponding

755 hypotheses and yellow denotes gene trees with no signal (polytomies). C. The signal obtained for

756 each gene genealogy compared to the phylogenetic hypotheses were plotted against the position

757 of the genes on the chromosome of Pantoea agglomerans. The same colour scheme is applied as

758 in the frequency histogram. All genes were located on the chromosome of P. agglomerans R190

759 and was distributed across the chromosome consisting of two contigs. Signal for the respective

760 nodes within the species phylogeny were distributed across the chromosome and no patterns of

761 shared signal were detected for groups of adjacent genes.

762 Figure 4. Seven single gene phylogenies determined with approximate maximum likelihood

763 (AML) analyses for genes identified as containing no signal (see File S4) and the maximum

764 likelihood (ML) phylogeny inferred from the combined sequence of these seven genes. A-G. The

765 AML phylogeny constructed from the protein sequences for the 30S ribosomal protein S18,

766 UDP-diphospho-muramoylpentapeptide beta-N-acetylglucosaminyltransferase, Prolyl-tRNA

767 synthetase, Glutamate 5-kinase, Cold shock-like protein $\operatorname{csp} C$, 30S ribosomal protein $\mathrm{S} 10$ and 
76830 S ribosomal protein S12, respectively. Taxa are numbered according to taxon descriptors in

769 panel H. H. The concatenated ML phylogeny constructed using RAxML v. 8.0.20 with the

770 appropriate amino acid model inferred using ProtTest v. 3.4 for each partition. All bootstrap

771 support values above $70 \%$ are indicated at nodes with dots. The phylogeny resembles the known

772 species phylogeny for Pantoea with the exception of some species relationships within the $P$.

773 agglomerans and $P$. rodasii lineages and the grouping of singleton taxa.

774 Figure 5. A circular diagram depicting the nucleotide concatenated alignment of all shared

775 genes. The outer track indicates the gene boundaries within the alignment, with tick marks

776 representing the length in nucleotides at 2,000 bp intervals. The second track indicates the

777 nucleotide positions within genes supporting conflicting topologies for the $P$. dispersa lineage to

778 species groupings observed in the concatenated species phylogeny. A total of 3,856 nucleotide

779 positions supported conflicting topologies for the $P$. dispersa lineage. The third track indicates

780 nucleotide positions supporting conflicting topologies for the P. ananatis lineage compared to

781 species groupings observed in the concatenated species phylogeny. For this lineage 1,764

782 nucleotide positions supported conflicting topologies for the $P$. ananatis lineage. The inner track

783 represents recombination breakpoints detected within gene boundaries for the concatenated

784 alignment. These breakpoints were supported by at least three of the five methods employed

785 (RDP, GENECONV, Chimaera, MaxChi and 3Seq) for detecting recombination. Stacked tiles

786 reflect the number of methods that were successful in detecting recombination events at those

787 regions, as well as multiple recombination events within the same region in various species (See

788 File S6).

789 Figure 6. The SH branch support for specific hypotheses in the trees constructed from the subset

790 datasets. Each hypothesis (Fig. 3A) was interrogated in each of the subset tree datasets, where 
$79120,50,60,70,80,90,100,110$ and 120 genes were used to construct ten randomised datasets for

792 each number of genes (File S7). The range indicated for each data point stretches from the lowest

793 branch support ( 0 in the case where the nodes were not recovered) to the highest branch support

794 (1 where the branch was fully supported) with the mean indicated with the data point. Regression

795 analyses were performed in Microsoft ExcelTM to fit the best regression model to the data. A.

796 Support for the three hypotheses depicted represent the monophyly of the three genera Erwinia

797 (hypothesis 1; green), Tatumella (hypothesis 2; red) and Pantoea (hypothesis 3; blue). All

798 subsets datasets recovered the nodes representing the monophyly of the genera, but in the case of

799 Pantoea, with less support in the replicates of the lower number of genes. B. The support for the

800 three test hypotheses i) separating the remainder of Pantoea from Pantoea sp. A4 (hypothesis 4;

801 green), ii) grouping $P$. septica, and the $P$. agglomerans and $P$. ananatis lineages together

802 (hypothesis 5; red) and iii) the grouping of $P$. cypripedii and Pantoea sp. At-9b with the $P$.

803 rodasii and $P$. dispersa lineages (hypothesis 9; blue). The node representing hypothesis 4 were

804 not recovered in two repeats of the 20 gene subsets. C. Hypothesis 6 depicts the sister grouping

805 of the $P$. agglomerans and $P$. ananatis lineages with the support associated with the node

806 depicted in green. The monophyletic grouping of the $P$. agglomerans lineage (hypothesis 7; red)

807 were not recovered in one 50 gene repeat, but were further fully supported in all repeats. The

808 grouping of the $P$. ananatis lineage was consistently recovered with full support (hypothesis 8 ;

809 blue). D. The support associated with the nodes depicting the sister relationship between the $P$.

810 rodasii and $P$. dispersa lineages (hypothesis 10; green). This node was not recovered in one of

811 the 20 gene repeats and one of the 50 gene repeats. Hypothesis 11 represents the monophyletic

812 grouping of the $P$. rodasii lineage, which was consistently recovered and well-supported with

813 branch support $>0.95$ (red). Branch support associated with the monophyletic grouping of the $P$. 
814 dispersa lineage often ranged from very low (0.24) to fully supported (1) in the 20, 50 and 60 815 gene repeats (hypothesis 12; blue). 


\section{Figure 1}

Comparison between the AML and MSCM phylogenies

A comparison between the two species trees based on the approaches employed in this study. Blue branches indicate differences in topology and branches with support of lower than 0.95 are indicated with an asterisk. A. The approximate maximum likelihood (AML) phylogeny constructed from the concatenated data matrix of the protein sequences of 1,357 genes, consisting of 337,780 amino acid columns. The phylogeny was constructed with FastTree v. 2.1 (Price et al., 2010) with the JTT (Jones et al., 1992) evolutionary model with CAT approximation. Simodaira-Hasegawa branch support values from 1,000 replicates were used. B. A species phylogeny using the multispecies coalescent model as implemented in ASTRAL v.5.6.3 (Mirarab et al., 2014) based on the individual phylogenies constructed from the protein sequences of 1,357 genes. The scale bar indicates one coalescent unit (Mirarab et al., 2014). Terminal branches are indicated as one coalescent unit, as branch lengths for taxa corresponding to species can only be calculated where multiple individuals per species are analysed. Shorter branches correspond to higher levels of incongruence and are generally associated with high levels of incomplete lineage sorting (ILS). Support values are determined based on Bayesian posterior probability values computed from the single gene tree quartet frequencies (Sayyari et al., 2016). 


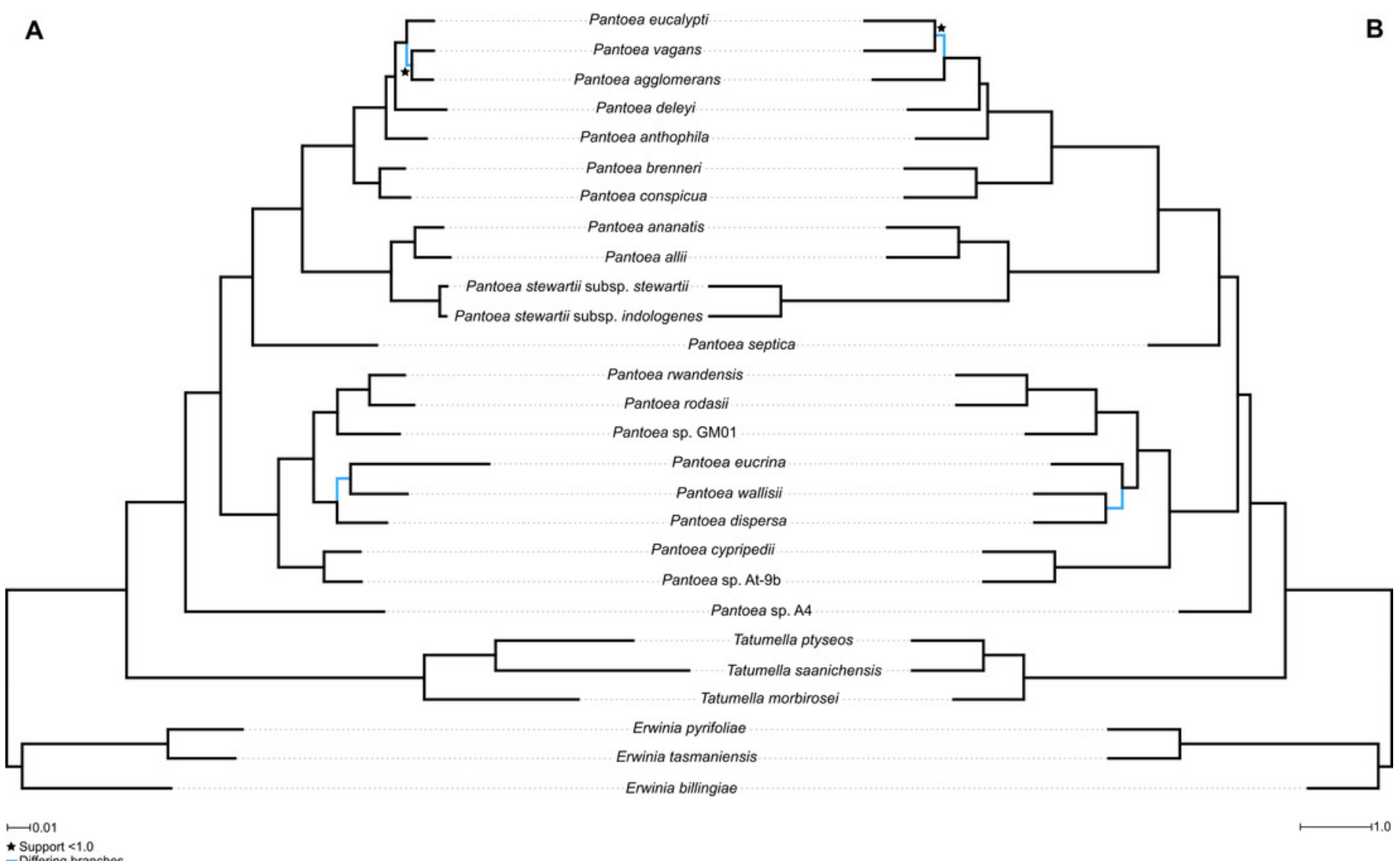


Figure 2

Neighbor-Net network from the concatenated nucleotide data

A Neighbor-Net network constructed from p-distances with equal angles for the concatenated nucleotide dataset. Overall, the configuration of the network is congruent with the existing species phylogeny for the genus Pantoea. Clear separation between the $P$. agglomerans and $P$. ananatis lineages were obtained and were also clearly distinct form the $P$. rodasii and $P$. dispersa lineages. Point A denotes where signal in conflict to the grouping of the $P$. ananatis lineage was determined, while point B denotes where signal in conflict to the grouping of the $P$. dispersa lineage was determined (see text for details).

*Note: Auto Gamma Correction was used for the image. This only affects the reviewing manuscript. See original source image if needed for review.

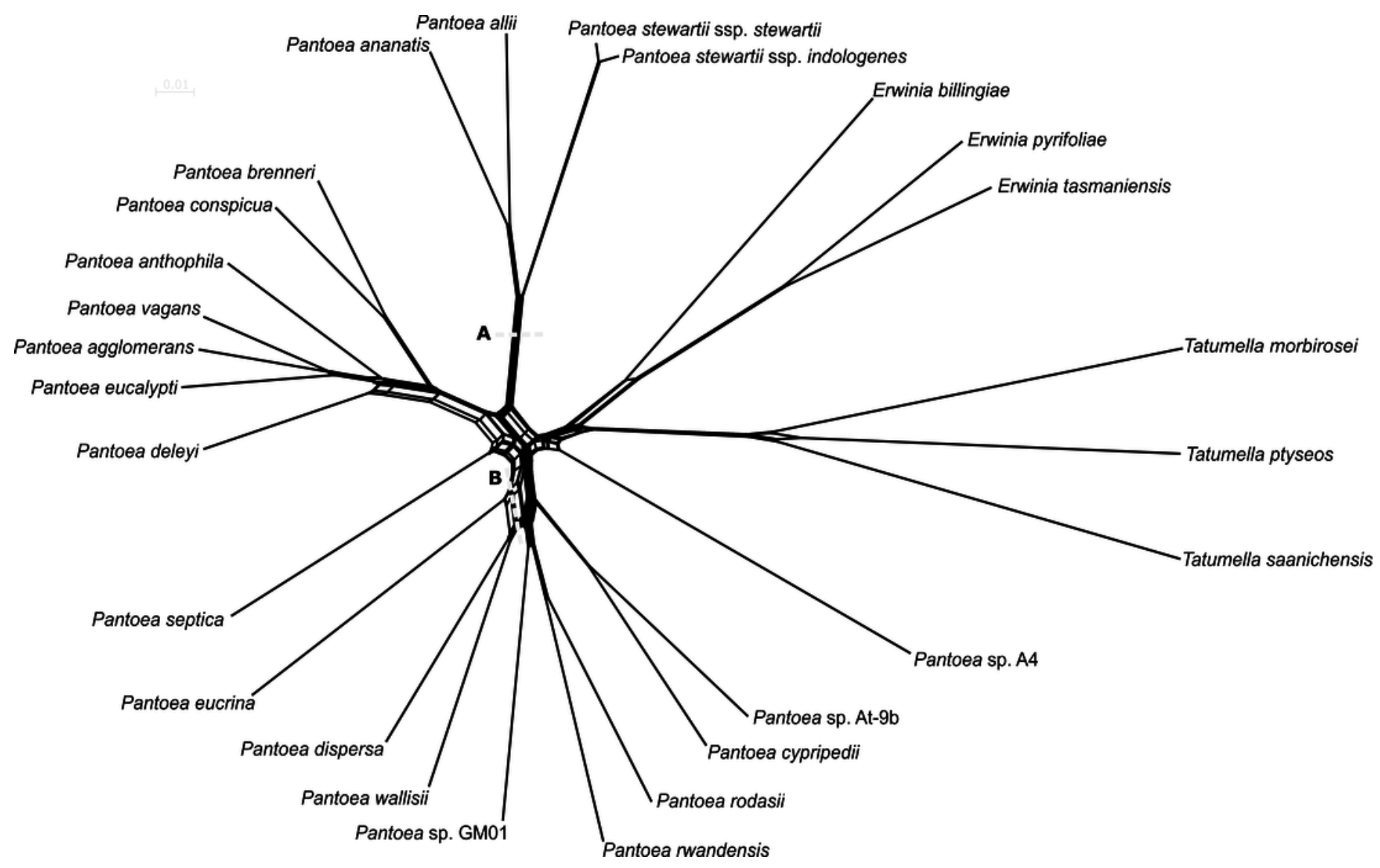




\section{Figure 3}

The summary of individual gene tree comparisons

An overview of the individual gene tree comparisons. A. The phylogenetic hypotheses evaluated during topology comparisons. Each number represents a specific hypothesis, where the monophyly of the group at each node was evaluated. An example is hypothesis 3 , where the overall monophyly of Pantoea was evaluated. B. A relative frequency histogram depicting the proportion of individual gene trees that support the phylogenetic hypotheses evaluated. Dark blue indicates genes that fully supported the monophyly of the corresponding hypothesis, while light blue indicates support for the monophyly of the hypothesis with additional taxa nested within the group. Red indicates gene trees that were incongruent with the corresponding hypotheses and yellow denotes gene trees with no signal (polytomies). C. The signal obtained for each gene genealogy compared to the phylogenetic hypotheses were plotted against the position of the genes on the chromosome of Pantoea agglomerans. The same colour scheme is applied as in the frequency histogram. All genes were located on the chromosome of $P$. agglomerans R190 and was distributed across the chromosome consisting of two contigs. Signal for the respective nodes within the species phylogeny were distributed across the chromosome and no patterns of shared signal were detected for groups of adjacent genes. 
A

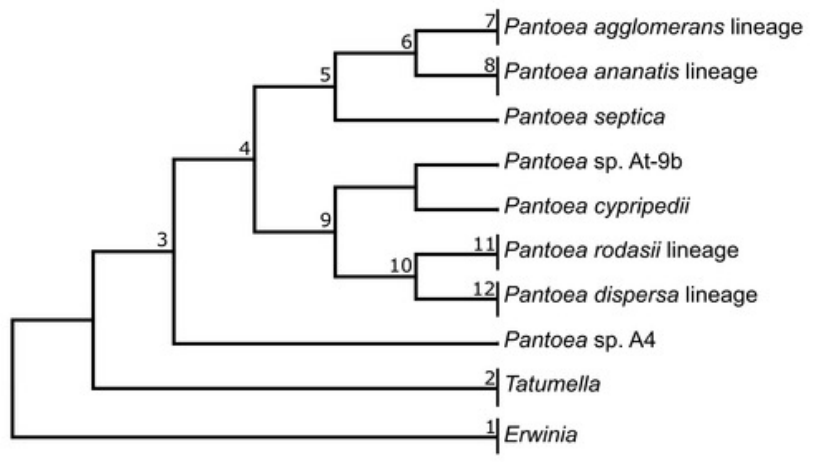

B
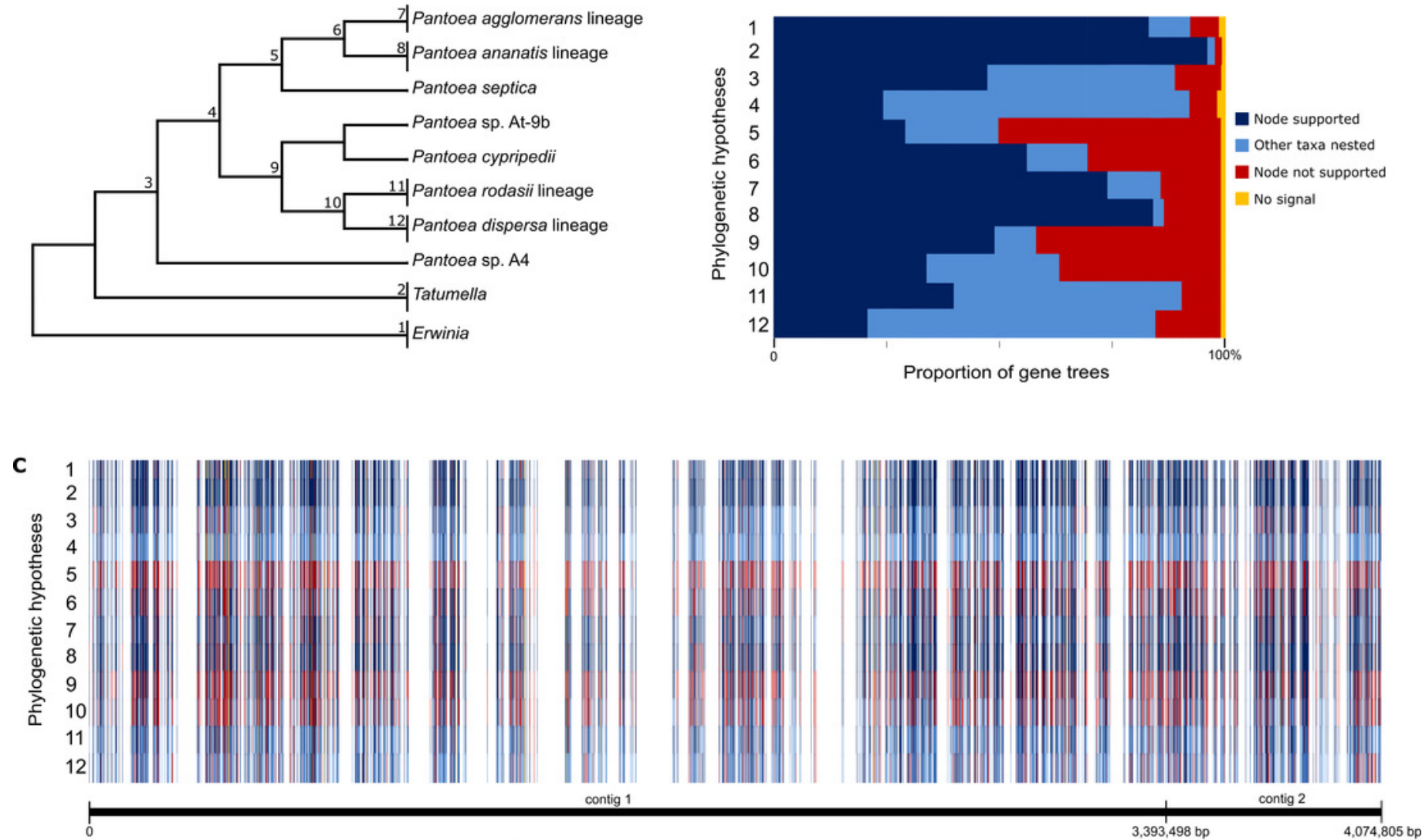

Position of signal on reference genome 


\section{Figure 4}

Summary of genes with limited to no signal

Seven single gene phylogenies determined with approximate maximum likelihood (AML) analyses for genes identified as containing no signal (see File S4) and the maximum likelihood (ML) phylogeny inferred from the combined sequence of these seven genes. A-G. The AML phylogeny constructed from the protein sequences for the $30 \mathrm{~S}$ ribosomal protein S18, UDP-diphospho-muramoylpentapeptide beta- $\mathrm{N}$-acetylglucosaminyltransferase, ProlyltRNA synthetase, Glutamate 5-kinase, Cold shock-like protein $\operatorname{cspC}$, 30S ribosomal protein S10 and 30 S ribosomal protein S12, respectively. Taxa are numbered according to taxon descriptors in panel $\mathbf{H}$. $\mathbf{H}$. The concatenated ML phylogeny constructed using RAXML V. 8.0.20 with the appropriate amino acid model inferred using ProtTest v. 3.4 for each partition. All bootstrap support values above $70 \%$ are indicated at nodes with dots. The phylogeny resembles the known species phylogeny for Pantoea with the exception of some species relationships within the $P$. agglomerans and $P$. rodasii lineages and the grouping of singleton taxa. 


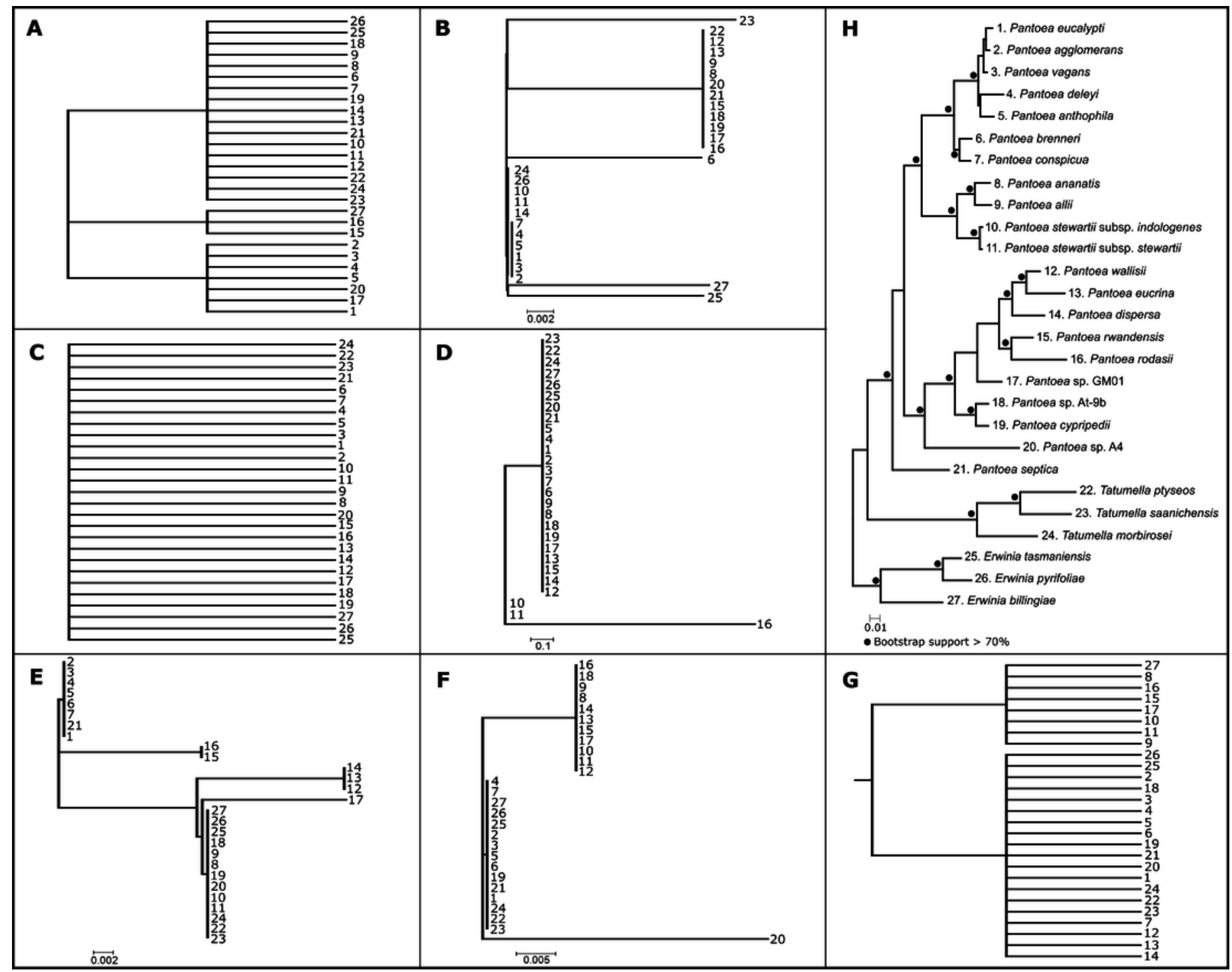




\section{Figure 5}

Conflicting signal and possible recombination breakpoints

A circular diagram depicting the nucleotide concatenated alignment of all shared genes. The outer track indicates the gene boundaries within the alignment, with tick marks representing the length in nucleotides at 2,000 bp intervals. The second track indicates the nucleotide positions within genes supporting conflicting topologies for the $P$. dispersa lineage to species groupings observed in the concatenated species phylogeny. A total of 3,856 nucleotide positions supported conflicting topologies for the $P$. dispersa lineage. The third track indicates nucleotide positions supporting conflicting topologies for the $P$. ananatis lineage compared to species groupings observed in the concatenated species phylogeny. For this lineage 1,764 nucleotide positions supported conflicting topologies for the $P$. ananatis lineage. The inner track represents recombination breakpoints detected within gene boundaries for the concatenated alignment. These breakpoints were supported by at least three of the five methods employed (RDP, GENECONV, Chimaera, MaxChi and 3Seq) for detecting recombination. Stacked tiles reflect the number of methods that were successful in detecting recombination events at those regions, as well as multiple recombination events within the same region in various species (See File S6). 


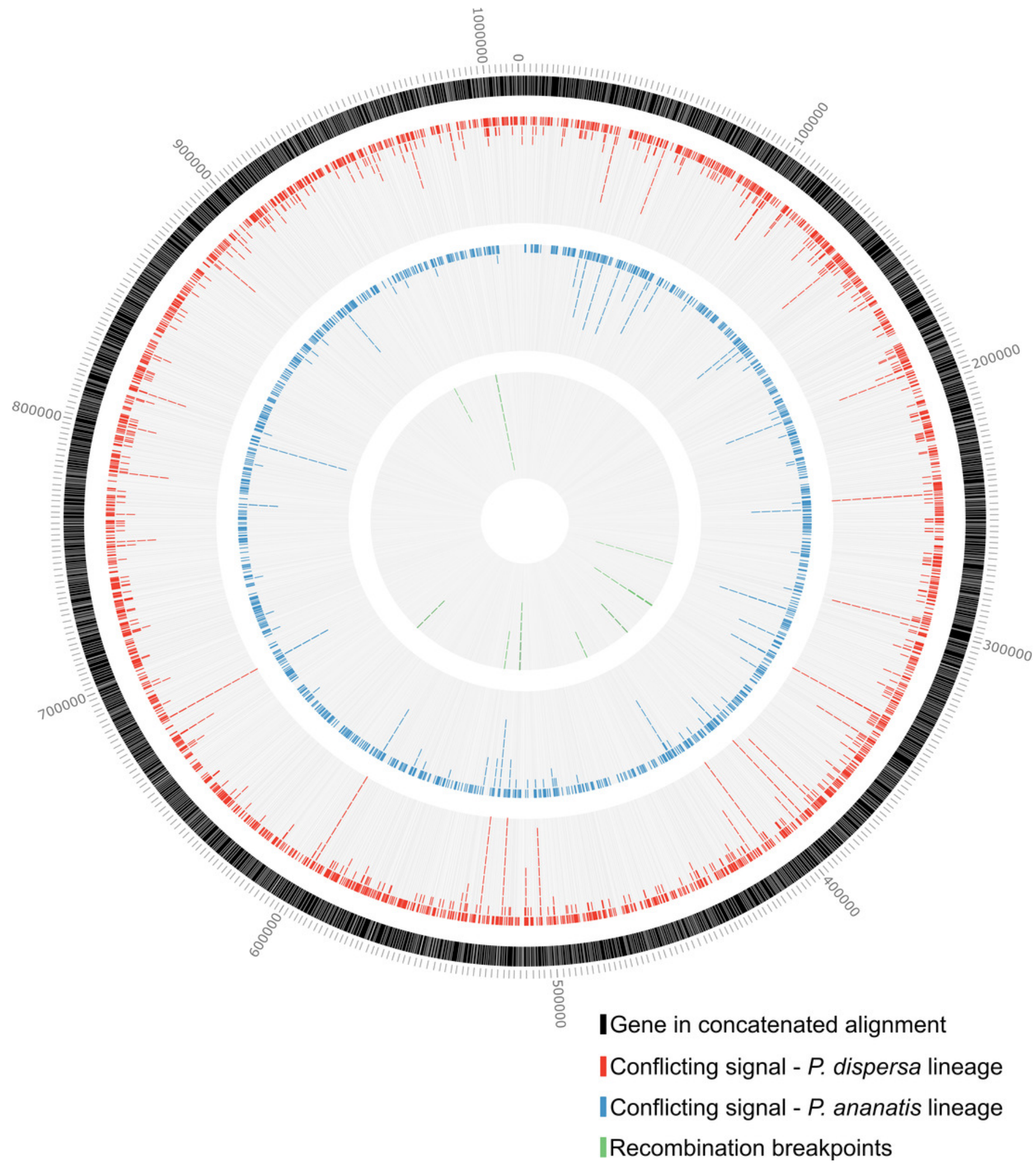




\section{Figure 6}

The SH branch support for specific hypotheses in the trees constructed from the subset datasets.

The SH branch support for specific hypotheses in the trees constructed from the subset datasets. Each hypothesis (Fig. 3A) was interrogated in each of the subset tree datasets, where $20,50,60,70,80,90,100,110$ and 120 genes were used to construct ten randomised datasets for each number of genes (File 57). The range indicated for each data point stretches from the lowest branch support ( 0 in the case where the nodes were not recovered) to the highest branch support ( 1 where the branch was fully supported) with the mean indicated with the data point. Regression analyses were performed in Microsoft ExcelTM to fit the best regression model to the data. A. Support for the three hypotheses depicted represent the monophyly of the three genera Erwinia (hypothesis 1; green), Tatumella (hypothesis 2; red) and Pantoea (hypothesis 3; blue). All subsets datasets recovered the nodes representing the monophyly of the genera, but in the case of Pantoea, with less support in the replicates of the lower number of genes. B. The support for the three test hypotheses i) separating the remainder of Pantoea from Pantoea sp. A4 (hypothesis 4; green), ii) grouping $P$. septica, and the $P$. agglomerans and $P$. ananatis lineages together (hypothesis 5; red) and iii) the grouping of $P$. cypripedii and Pantoea sp. At-9b with the $P$. rodasii and $P$. dispersa lineages (hypothesis 9 ; blue). The node representing hypothesis 4 were not recovered in two repeats of the 20 gene subsets. C. Hypothesis 6 depicts the sister grouping of the $P$. agglomerans and $P$. ananatis lineages with the support associated with the node depicted in green. The monophyletic grouping of the $P$. agglomerans lineage (hypothesis 7; red) were not recovered in one 50 gene repeat, but were further fully supported in all repeats. The grouping of the $P$. ananatis lineage was consistently recovered with full support (hypothesis 8; blue). D. The support associated with the nodes depicting the sister relationship between the $P$. rodasii and $P$. dispersa lineages (hypothesis 10; green). 
This node was not recovered in one of the 20 gene repeats and one of the 50 gene repeats. Hypothesis 11 represents the monophyletic grouping of the $P$. rodasii lineage, which was consistently recovered and well-supported with branch support $>0.95$ (red). Branch support associated with the monophyletic grouping of the $P$. dispersa lineage often ranged from very low (0.24) to fully supported (1) in the 20,50 and 60 gene repeats (hypothesis 12; blue).
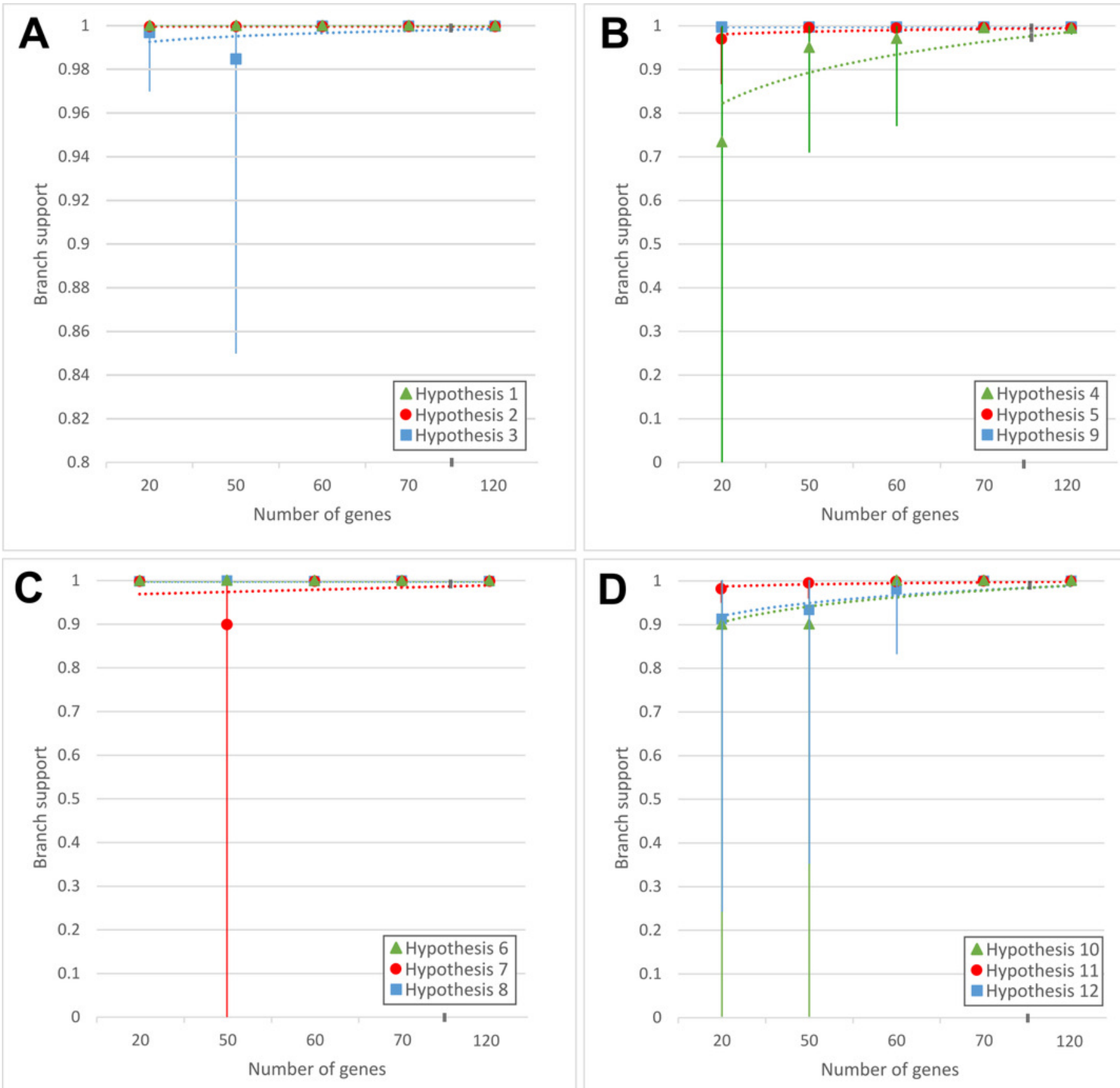
Table $\mathbf{1}$ (on next page)

Genome sequences utilised in this study 
1 Table 1. Genome sequences utilised in this study

\begin{tabular}{|c|c|c|c|c|}
\hline Genus & Species & Strain $^{\text {a }}$ & Accession number ${ }^{b}$ & Reference \\
\hline \multirow[t]{21}{*}{ Pantoea } & Pantoea agglomerans & R 190 & JNGC00000000.1 & Lim et al., 2014 \\
\hline & Pantoea allii & LMG $24248^{\mathrm{T}}$ & MLFE00000000.1 & Palmer et al., 2017 \\
\hline & Pantoea ananatis & LMG $2665^{\mathrm{T}}$ & JMJJ00000000.1 & De Maayer et al., 2014 \\
\hline & Pantoea anthophila & $11-2$ & JXXL00000000.1 & Wan et al., 2015 \\
\hline & Pantoea brenneri & LMG $5343^{\mathrm{T}}$ & MIEI00000000.1 & Palmer et al., 2017 \\
\hline & Pantoea conspicua & LMG $24534^{\mathrm{T}}$ & MLFN00000000.1 & Palmer et al., 2017 \\
\hline & Pantoea cypripedii & LMG $2657^{\mathrm{T}}$ & MLJI00000000.1 & Palmer et al., 2017 \\
\hline & Pantoea deleyi & LMG $24200^{\mathrm{T}}$ & MIPO00000000.1 & Palmer et al., 2017 \\
\hline & Pantoea dispersa & EGD-AAK13 & AVSS00000000.1 & - \\
\hline & Pantoea eucalypti & $\mathrm{aB}$ & AEDL00000000.1 & - \\
\hline & Pantoea eucrina & LMG $2781^{\mathrm{T}}$ & MIPP00000000.1 & Palmer et al., 2017 \\
\hline & Pantoea rodasii & LMG $26273^{\mathrm{T}}$ & MLFP00000000.1 & Palmer et al., 2017 \\
\hline & Pantoea rwandensis & LMG $26275^{\mathrm{T}}$ & MLFR00000000.1 & Palmer et al., 2017 \\
\hline & Pantoea septica & LMG $5345^{\mathrm{T}}$ & MLJJ00000000.1 & Palmer et al., 2017 \\
\hline & $\begin{array}{l}\text { Pantoea stewartii ssp. } \\
\text { stewartii }\end{array}$ & DC 283 & AHIE00000000.1 & - \\
\hline & $\begin{array}{l}\text { Pantoea stewartii ssp. } \\
\text { indologenes }\end{array}$ & LMG $2632^{\mathrm{T}}$ & JPKO00000000.1 & - \\
\hline & Pantoea vagans & C9-1 & $\begin{array}{l}\text { CP001894.1, } \\
\text { CP001893.1, } \\
\text { CP001894.1 }\end{array}$ & Smits et al., 2010 \\
\hline & Pantoea wallisii & LMG $26277^{\mathrm{T}}$ & MLFS00000000.1 & Palmer et al., 2017 \\
\hline & Pantoea sp. & At-9b & $\begin{array}{l}\text { CP002433.1, } \\
\text { CP002434.1, } \\
\text { CP002435.1, } \\
\text { CP002436.1, } \\
\text { CP002437.1, } \\
\text { CP002438.1 }\end{array}$ & Suen et al., 2010 \\
\hline & Pantoea sp. & A4 & ALXE00000000.1 & Hong et al., 2012 \\
\hline & Pantoea sp. & GM01 & AKUI00000000.1 & Brown et al., 2012 \\
\hline \multirow[t]{3}{*}{ Tatumella } & Tatumella morbirosei & LMG $23360^{\mathrm{T}}$ & CM003276.1 & - \\
\hline & Tatumella ptyseos & ATCC $33301^{\mathrm{T}}$ & ATMJ00000000.1 & - \\
\hline & Tatumella saanichensis & NML 06-3099 ${ }^{\mathrm{T}}$ & ATMI00000000.1 & Tracz et al., 2015 \\
\hline \multirow[t]{3}{*}{ Erwinia } & Erwinia billingiae & NCPPB $661^{\mathrm{T}}$ & $\begin{array}{l}\text { FP236843.1,FP236826.1, } \\
\text { FP236830.1 }\end{array}$ & Kube et al., 2010 \\
\hline & Erwinia pyrifoliae & DSM $12163^{\mathrm{T}}$ & $\begin{array}{l}\text { FN392235.1, } \\
\text { FN392236.1, } \\
\text { FN392237.1 }\end{array}$ & Kube et al., 2010 \\
\hline & Erwinia tasmaniensis & Et $1-99^{\mathrm{T}}$ & $\begin{array}{l}\text { CU468135.1, } \\
\text { CU468128.1, } \\
\text { CU468130.1, } \\
\text { CU468131.1, } \\
\text { CU468132.1, } \\
\text { CU468133.1 }\end{array}$ & Kube et al., 2008 \\
\hline
\end{tabular}

$2{ }^{\text {a Superscript }}{ }^{\mathrm{T}}$ indicates type strains for the species

3 b All numbers refer to Genbank assembly accession numbers (http://www.ncbi.nlm.nih.gov/; accessed $428 / 2 / 2017)$ 


\section{Table 2 (on next page)}

Summary of gene subset tests 
1 Table 2. Summary of gene subset tests ${ }^{a}$

\begin{tabular}{|c|c|c|c|c|}
\hline $\begin{array}{c}\text { Number of } \\
\text { genes in subset }\end{array}$ & Replicate & Length (bp) & $\begin{array}{l}\text { Backbone nodes } \\
\text { support range }\end{array}$ & $\begin{array}{l}\text { Leaf nodes } \\
\text { support range }\end{array}$ \\
\hline \multirow[t]{10}{*}{120 genes } & 1 & 40,069 & 1.00 & $0.50-1.00$ \\
\hline & 2 & 36,614 & 1.00 & $0.37-1.00$ \\
\hline & 3 & 39,663 & 1.00 & $0.93-1.00$ \\
\hline & 4 & 38,050 & 1.00 & $0.43-1.00$ \\
\hline & 5 & 37,931 & 1.00 & $0.46-1.00$ \\
\hline & 6 & 40,260 & 1.00 & $0.57-1.00$ \\
\hline & 7 & 39,776 & 1.00 & $0.82-1.00$ \\
\hline & 8 & 40,385 & 1.00 & $0.86-1.00$ \\
\hline & 9 & 42,793 & 1.00 & $0.67-1.00$ \\
\hline & 10 & 39,328 & 1.00 & $0.59-1.00$ \\
\hline \multirow[t]{10}{*}{110 genes } & 1 & 35,298 & 1.00 & $0.95-1.00$ \\
\hline & 2 & 35,349 & 1.00 & $0.69-1.00$ \\
\hline & 3 & 36,798 & 1.00 & $0.86-1.00$ \\
\hline & 4 & 38,800 & 1.00 & $0.40-1.00$ \\
\hline & 5 & 35,445 & 1.00 & $0.73-1.00$ \\
\hline & 6 & 38,042 & 1.00 & $0.71-1.00$ \\
\hline & 7 & 40,172 & $0.99-1.00$ & $0.38-1.00$ \\
\hline & 8 & 39,865 & 1.00 & $0.18-1.00$ \\
\hline & 9 & 40,737 & 1.00 & $0.78-1.00$ \\
\hline & 10 & 40,745 & 1.00 & $0.54-1.00$ \\
\hline \multirow[t]{10}{*}{100 genes } & 1 & 33,340 & 1.00 & $0.78-1.00$ \\
\hline & 2 & 30,822 & $0.99-1.00$ & $0.47-1.00$ \\
\hline & 3 & 33,433 & 1.00 & $0.65-1.00$ \\
\hline & 4 & 30,707 & 1.00 & $0.90-1.00$ \\
\hline & 5 & 31,340 & 1.00 & $0.58-1.00$ \\
\hline & 6 & 31,798 & 1.00 & $0.87-1.00$ \\
\hline & 7 & 29.562 & 1.00 & $0.64-1.00$ \\
\hline & 8 & 30,773 & 1.00 & $0.06-1.00$ \\
\hline & 9 & 34,064 & 1.00 & $0.88-1.00$ \\
\hline & 10 & 35,550 & 1.00 & $0.68-1.00$ \\
\hline \multirow[t]{10}{*}{90 genes } & 1 & 31,353 & 1.00 & $0.68-1.00$ \\
\hline & 2 & 29,307 & $0.99-1.00$ & $0.91-1.00$ \\
\hline & 3 & 31,941 & 1.00 & $0.94-1.00$ \\
\hline & 4 & 31,890 & 1.00 & $0.77-1.00$ \\
\hline & 5 & 29,695 & 1.00 & $0.84-1.00$ \\
\hline & 6 & 30,564 & 1.00 & $0.37-1.00$ \\
\hline & 7 & 25,162 & 1.00 & $0.78-1.00$ \\
\hline & 8 & 30,745 & 1.00 & $0.48-1.00$ \\
\hline & 9 & 28,146 & 1.00 & $0.55-1.00$ \\
\hline & 10 & 28,883 & 1.00 & $0.81-1.00$ \\
\hline \multirow[t]{6}{*}{80 genes } & 1 & 24,020 & 1.00 & $0.35-1.00$ \\
\hline & 2 & 23,065 & 1.00 & $0.73-1.00$ \\
\hline & 3 & 25,922 & $0.99-1.00$ & $0.86-1.00$ \\
\hline & 4 & 27,877 & 1.00 & $0.53-1.00$ \\
\hline & 5 & 25,288 & $0.99-1.00$ & $0.70-1.00$ \\
\hline & 6 & 22,551 & $0.98-1.00$ & $0.69-1.00$ \\
\hline
\end{tabular}




\begin{tabular}{|c|c|c|c|c|}
\hline & 7 & 26,417 & 1.00 & $0.59-1.00$ \\
\hline & 8 & 27,008 & 1.00 & $0.72-1.00$ \\
\hline & 9 & 25,156 & 1.00 & $0.30-1.00$ \\
\hline & 10 & 25,498 & $0.98-1.00$ & $0.77-1.00$ \\
\hline \multirow[t]{10}{*}{70 genes } & 1 & 22,011 & $0.99-1.00$ & $0.16-1.00$ \\
\hline & 2 & 24,373 & 1.00 & $0.54-1.00$ \\
\hline & 3 & 24,420 & 1.00 & $0.45-1.00$ \\
\hline & 4 & 20,887 & 1.00 & $0.82-1.00$ \\
\hline & 5 & 22,286 & $0.99-1.00$ & $0.11-1.00$ \\
\hline & 6 & 22,702 & 1.00 & $0.27-1.00$ \\
\hline & 7 & 23,787 & $0.99-1.00$ & $0.83-1.00$ \\
\hline & 8 & 19,750 & 1.00 & $0.21-1.00$ \\
\hline & 9 & 23,770 & $0.99-1.00$ & $0.68-1.00$ \\
\hline & 10 & 21,613 & 1.00 & $0.31-1.00$ \\
\hline \multirow[t]{10}{*}{60 genes } & 1 & 18,755 & $0.99-1.00$ & $0.92-1.00$ \\
\hline & 2 & 21,310 & $0.99-1.00$ & $0.89-1.00$ \\
\hline & 3 & 21,745 & $0.99-1.00$ & $0.77-1.00$ \\
\hline & 4 & 19,210 & 1.00 & $0.83-1.00$ \\
\hline & 5 & 19,495 & 1.00 & $0.83-1.00$ \\
\hline & 6 & 18,550 & $0.83-1.00$ & $0.69-1.00$ \\
\hline & 7 & 20,389 & 1.00 & $0.58-1.00$ \\
\hline & 8 & 20,475 & $0.77-1.00$ & $0.47-1.00$ \\
\hline & 9 & 17,331 & 1.00 & $0.01-1.00$ \\
\hline & 10 & 23,324 & $0.97-1.00$ & $0.40-1.00$ \\
\hline \multirow[t]{10}{*}{50 genes } & 1 & 14,890 & $1.00 *$ & $0.31-1.00$ \\
\hline & 2 & 18,079 & 1.00 & $0.27-1.00$ \\
\hline & 3 & 14,701 & 1.00 & $0.81-1.00$ \\
\hline & 4 & 13,983 & $0.71-1.00$ & $0.00-1.00$ \\
\hline & 5 & 19,059 & 1.00 & $0.40-1.00$ \\
\hline & 6 & 18,412 & $0.99-1.00$ & $0.86-1.00$ \\
\hline & 7 & 18,880 & 1.00 & $0.59-1.00$ \\
\hline & 8 & 14,411 & $0.85-1.00$ & $0.33-1.00$ \\
\hline & 9 & 14,942 & $0.81-1.00$ & $0.67-1.00$ \\
\hline & 10 & 14,531 & 1.00 & $0.28-1.00$ \\
\hline \multirow[t]{10}{*}{20 genes } & 1 & 5,966 & $0.87-1.00$ & $0.74-1.00$ \\
\hline & 2 & 5,834 & $0.99-1.00 *$ & $0.64-1.00$ \\
\hline & 3 & 6,859 & $0.98-1.00$ & $0.58-1.00$ \\
\hline & 4 & 7,152 & $0.97-1.00 *$ & $0.00-1.00$ \\
\hline & 5 & 5,560 & $0.99-1.00$ & $0.26-1.00$ \\
\hline & 6 & 6,316 & $0.48-1.00$ & $0.71-1.00$ \\
\hline & 7 & 6,210 & $0.93-1.00$ & $0.26-1.00$ \\
\hline & 8 & 6,517 & $0.99-1.00$ & $0.22-1.00$ \\
\hline & 9 & 6,649 & $0.95-1.00$ & $0.06-1.00$ \\
\hline & 10 & 6,436 & $0.90-1.00$ & $0.53-1.00$ \\
\hline
\end{tabular}

$2 \quad{ }^{\text {a }}$ See File S3

3 b One backbone node is not recovered in the phylogenies marked with an asterisk. 